\title{
Effect of Confinement on Surface Modification for Laser Peen Forming without Protective Coating
}

\author{
Ninggang Shen ${ }^{1}$, Hongtao Ding ${ }^{1, *}$, Qinghua Wang ${ }^{1}$, Hua Ding ${ }^{2}$ \\ ${ }^{1}$ Department of Mechanical \& Industrial Engineering, University of Iowa, Iowa City, IA 52242, USA \\ ${ }^{2}$ School of Automotive and Traffic Engineering, Jiangsu University, Zhenjiang, 212013, China
}

\begin{abstract}
In this study, multiple laser peen forming (LPF) experiments were conducted for micro-bending Aluminum alloy 1060, OHFC Copper, and commercially pure Titanium specimens under confinement media of water and air. Surface morphological and compositional changes as well as the mechanical properties of the treated specimens were experimentally analyzed to determine the effect of confinement during LPF. Significantly different surface morphological characteristics were captured and quantitatively analyzed. Clear feed mark patterns were found to be caused by the laser pulse feed for all materials under air confinement. Under water confinement, specimen surfaces appeared in a tarnished, featureless surface texture without showing any feed marks. The mechanisms of laser-material interaction during LPF were studied for various materials under different confinement media. The surface chemical composition change was investigated on the surface and in depth. More chemical composition change was observed under air confinement than under water, while it was limited within the topmost 10-30 nm under both water and air confinement.
\end{abstract}

\section{Keywords}

Laser peen forming, Surface integrity, Confinement medium, Phase explosion, Chemical composition.

*Corresponding author: hongtao-ding@uiowa.edu, +1-319-335-5674 


\section{Introduction}

The need of precise and non-contact adjustment has been increased in both industry and laboratory to change the shape of microscale structures and components [1]. Novel laser peen forming (LPF) processes have been developed based on the mechanism of laser shock peening in order to fulfill these demands [2]. Recent research progress has provided a sound understanding of the process principle for laser shock peening. During the laser shock peening process, a plasma plume is generated on the target metal surface with the irradiation of high-energy laser with a pulse duration in nanoseconds. The hot and dense plasma tends to expand itself rapidly. Due to the effect of confinement medium, usually air or water, the expansion of plasma into atmosphere is confined. A strong compressive shock wave is thus generated against the target metal surface with a peak pressure usually greater than $5 \mathrm{GPa}$ during a time duration of 0.6-30 nanoseconds [3,4]. This laser-induced strong shock compression is used to form metal materials during LPF processes [5]. The development of the LPF process can enable die-free design flexibility, production of complex shapes, thick plate forming, and spatial forming of a sheet metal component [6,7]. For instance, a laser peen micro-bending method has been applied to accurately adjust micro-components for micro-electro-mechanical system [8-11] or optical devices [12].

A lot of research effort has been devoted to understand the forming mechanism for the LPF process. Hackel and Harris [13] developed an approach and experimental set-up for shape and contour forming of a metal workpiece to investigate the forming process capability of LPF. Hu et al. [6] experimentally studied the two-direction bending mechanism of nanosecond-pulsed laser bending. From their experimental analysis, specimen thickness and size of the plastic deformation zone below the peened surface have been identified as the critical parameters for the bending mechanisms. Vasu et al. experimentally demonstrated the effect of pre-existing curvature on the target surface on the plastic zone and residual stress distribution, and investigated the mechanism with numerical model [14,15]. Ding et al. [16] performed an experimental study of laser micro-bending for aluminum alloy 1060 using a Nd:YAG laser with a wavelength of $1064 \mathrm{~nm}$ and determined the transitional specimen thickness between concave and convex bending mechanisms. Pence et al. [17] further investigated the bending mechanisms by modeling the physics of laser-matter interaction during LPF. Their study helped control the geometric tolerancing for micro-bending of specimen with various thicknesses. The capability of concavely and convexly bending of sheet metals by LPF has been approved beneficial in both micro- and macro-scale applications [18].

Surface strength enhancement and microstructural refinement due to laser shock peening has been studied extensively. It was reported that grains in the surface layer can be considerably refined to ultra- 
fine or nano-grains due to the dense dislocation structure formed during the severe plastic deformation induced by the strong shock compression [19]. The significantly refined microstructure, as well as the induced compressive residual stress, can improves the resistances to fatigue [20-22]. Corrosion resistance was found to be improved by introducing a protective passive oxide film on the target surface, e.g. $\mathrm{Al}_{2} \mathrm{O}_{3}$ for aluminum alloys $[23,24]$.

It is also critical to investigate the induced surface modifications during LPF and understand their effects on component performance. Gu et al. [25] recently performed an experimental study of LPF of aluminum alloy 1060 under water confinement and found that the average surface roughness of adjusted surface was reduced to about $7.6 \mu \mathrm{m}$ from the untreated surface roughness of $10 \mu \mathrm{m}$. The surface morphology change of laser shock peening without protective coating has been reported for bulk metal materials under water confinement. Kalainathan et al. [26] and Karthik et al. [27] characterized the surface roughness change for surface enhancement of various bulk stainless steels. It was found that the surface roughness increased by more pronounced peening patterns on the surface, and this increase directly correlated with the laser pulse density during the process. Trdan et al. [28] performed a laser shock peening study on aluminum alloy 6082-T651 without protective coating and found that greater micro-crater depth and area were caused by a high laser pulse density.

Nonetheless, the effects of confinement medium on morphological and compositional changes during LPF have been rarely investigated. Because of the high temperature and pressure surroundings induced by the laser irradiation, surface chemical composition is likely to be changed; however, it has not been studied from the previous research on LPF or laser shock peening. In addition, the material effect on the modified surfaces has not been well understood for the LPF process.

In this work, surface integrity issues in terms of surface morphological modification and chemical composition are evaluated based on micro-bending experiments for three metal materials using a nanosecond pulsed laser. The treated specimens are investigated and analyzed in terms of surface morphology, surface microhardness, and chemical composition for all materials in both air and water confinements. The effect of confinement media is discussed for the different surface morphologies of various materials. A theoretical analysis is conducted to understand the surface morphology changes induced by different laser-material mechanisms.

\section{LPF Experiments}

The configuration of laser peen micro-bending experiment is illustrated in Fig. 1a. A Q-Switched Nd:YAG laser was used in the experiment. The wavelength was $1064 \mathrm{~nm}$, the repetition rate was $10 \mathrm{~Hz}$, and pulse duration was 6-8 ns. The laser pulse was delivered along the optical path to the target surface as 
shown in Fig. 1a. The laser pulse energy, $E$, was set two levels of 0.3 and $0.4 \mathrm{~J}$. The diameter of the laser spots, $D$, was $1.5 \mathrm{~mm}$. The specimen was fixed on a workpiece holder in a water tank mounted on X-Y motion stages. During the experiment, the laser scanned the top surface of specimen in a raster pattern as illustrated in Fig. 1b. A constant scanning speed and increment width of the raster $(X)$ were applied, which were $2.8 \mathrm{~mm} / \mathrm{s}$ and $0.35 \mathrm{~mm}$, respectively. Three workpiece materials were tested, i.e., Aluminum Alloy 1060 (Al), OHFC Copper (Cu), and commercially pure Titanium (cpTi). The sheet metal of all materials with various thicknesses were cut into a rectangular coupon of $76 \mathrm{~mm}$ in length and $13 \mathrm{~mm}$ in width. The original arithmetic average surface roughness, $R_{a}$, of the original coupon surfaces prior to laser treatment was $0.08 \mu \mathrm{m}, 1.63 \mu \mathrm{m}$, and $0.26 \mu \mathrm{m}$ for $\mathrm{Al}, \mathrm{cpTi}$, and $\mathrm{Cu}$ specimens. Water and air were used as the confinement media. When using water as the confinement medium, the tank was filled with distilled water to the level about $10 \mathrm{~mm}$ higher than the top coupon surface, while the tank was empty when using air as the confinement medium.

a) Experimental Setup

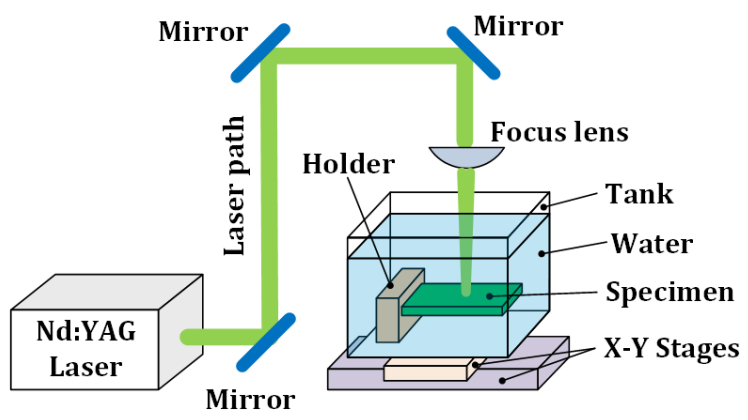

b) Scanning Paths

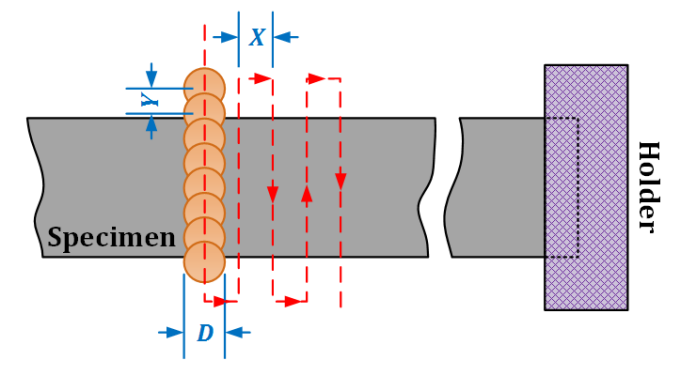

Fig. 1 Laser Peen Micro-Bending Configurations: a) Experimental Setup; b) Laser Scanning Paths.

\section{Mechanical Properties}

Surface microhardness of the specimens was measured using a LECO LM-300AT Vickers microindentation hardness tester. Before the LPF experiments, the average hardness of the as-received specimens were $125.6 \mathrm{HV}, 62.8 \mathrm{HV}$, and $255.6 \mathrm{HV}$ for $\mathrm{Al}, \mathrm{Cu}$, and $\mathrm{cpTi}$, respectively. After LPF, 
multiple microhardness indentation measurements were conducted directly on the scanned surface. The averages increased to $173.3 \mathrm{HV}$ (by $38.0 \%$ ), $114.8 \mathrm{HV}$ (by $82.8 \%$ ), and $374.5 \mathrm{HV}$ (by $46.5 \%$ ) for $\mathrm{Al}, \mathrm{Cu}$, and cpTi, respectively, using water as the confinement medium. Thus, the material hardness as well as mechanical strength was increased significantly in the peened area for all three materials. The residual stress of the $\mathrm{Al}$ specimens scanned by laser pulses of $0.4 \mathrm{~J}$ under water confinement were measured by $\mathrm{X}$ ray diffraction using the multi-angle $\sin 2 \psi$ technique. Table 1 shows the residual stress measurements in two components of $\sigma_{x x}$ (transverse) and $\sigma_{y y}$ (longitudinal) on the specimen surface. High compressive residual stresses (less than $-200 \mathrm{MPa}$ ) were obtained along both components. The minimum of the residual stress in longitudinal direction was $-276 \mathrm{MPa}$ for the $0.75 \mathrm{~mm}$ thick specimen, while that in transverse direction was $-342 \mathrm{MPa}$ for $1.75 \mathrm{~mm}$ thick specimen.

Table 1. Residual stress results ( $\mathrm{E}=0.4 \mathrm{~J}$, under water).

\begin{tabular}{|c|c|c|}
\hline Specimens & $\boldsymbol{\sigma}_{\mathbf{x x}}(\mathbf{M P a})$ & $\boldsymbol{\sigma}_{\mathbf{y y}}(\mathbf{M P a})$ \\
\hline Al, 0.7 $\mathbf{~ m m}$ thick & $-248 \pm 31$ & $-276 \pm 56$ \\
\hline Al, $\mathbf{1 . 7 5} \mathbf{~ m m}$ thick & $-342 \pm 104$ & $-207 \pm 23$ \\
\hline
\end{tabular}

\section{Surface Morphology Analysis}

Fig. 2 shows the raster-scanned (five scan tracks) surface of $\mathrm{Cu}$ specimens under the optical microscope. The specimens were laser treated in air and water by $0.4 \mathrm{~J}$ laser pulses. A zoom-in view are also shown to reveal more details. For the surface scanned in air as shown in Fig. 2a, each scan track can be easily recognized by its appearance. These scan tracks were labeled in scanning sequences. The remaining feed marks from the previous 4 tracks are noticeable, even though most of the areas were removed by the next lateral scans. Compared with the previous four scan tracks, the feed marks from the last scan (Scan-5) were very obvious. The feed marks appear darker in color than the areas in between the adjacent feed marks, where a fairly fresh copper color and metallic luster can be seen. The different appearances in color and luster likely resulted from different degrees of oxidization during or after scanning in air. Fig. $2 \mathrm{~b}$ shows the scanned $\mathrm{Cu}$ specimen surface under water confinement. Without showing any feed mark or different color for an individual scan track, the specimen surface appears in a tarnished, featureless surface texture. Similar textures were observed for the other materials as well, i.e. clear feed mark patterns for specimens in air and featureless texture for specimens in water, respectively. 


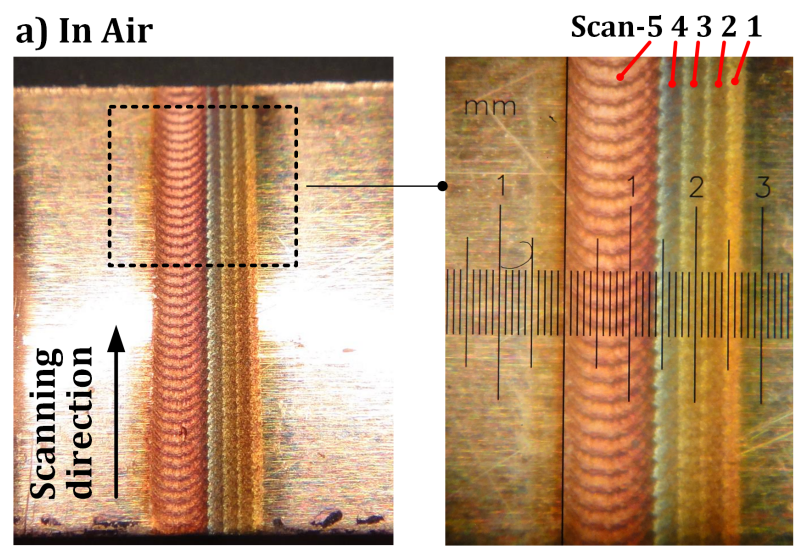

\section{b) In Water}

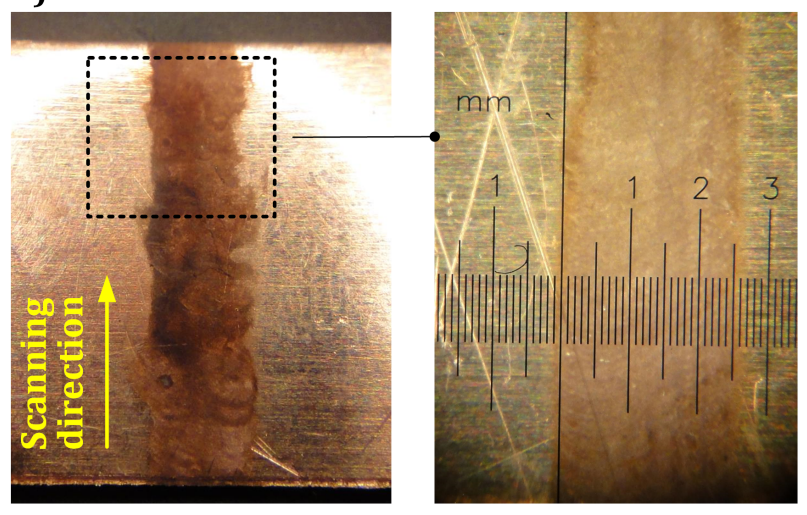

Fig. 2 Comparison of raster-scanned $\mathrm{Cu}$ surface after LPF. a) in air; b) in water.

\subsection{Surface Produced in Air}

To investigate the height profile of feed marks, the three-dimensional (3D) surface profiles of the scanned tracks for each material were firstly captured by a Veeco Wyko NT1100 white light interferometer. Fig. 3 shows the 3D profiles of the laser-scanned tracks of cpTi and $\mathrm{Cu}$ treated in air. The feed mark patterns can be seen for both materials, but the height distribution characteristics were significantly different. For the cpTi surface, the feed marks are outstanding wavefront-like ridges with a uniform spatial increment between shots. The area between the adjacent ridges were relatively smooth. The measured height of the ridges were about $10 \mu \mathrm{m}$. For $\mathrm{Cu}$, the surface was rough without the sharp ridges as those on a scanned cpTi surface. In the height profiles for the $\mathrm{Cu}$ and $\mathrm{Al}$ specimens, the dark areas surrounding the incontinuous spikes were regions without picking any data. The missing data could be caused by high surface roughness or porous surface morphology. 

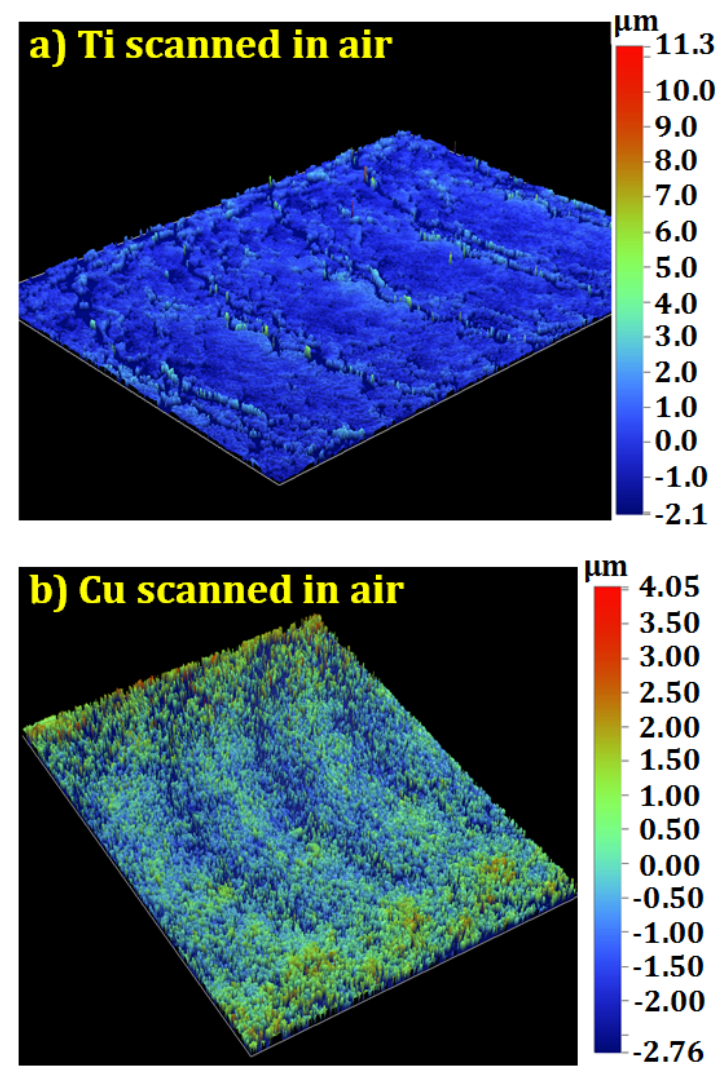

Fig. 3 3D scanned surface profiles in air: a) cpTi; b) $\mathrm{Cu}$.

To improve the accuracy of surface profile measurement, a Taylor Hobson ${ }^{\circledR}$ Surtronic 25 surface roughness profilometer was applied to obtain the two-dimensional (2D) surface profiles using a diamond stylus with a tip radius of $5 \mu \mathrm{m}$. The surface profile was obtained by a $2.5 \mathrm{~mm}$ evaluation length. The resolution of height profile was $0.01 \mu \mathrm{m}$ and the sampling increment is $0.5 \mu \mathrm{m}$. The measurement data were processed and visualized by the analysis software of Talyprofile Lite. Fig. 4 presents the comparison of the scanned surface profile of all three materials in air. For both $\mathrm{Al}$ and cpTi, there were many spikes along the scanned track. Among all the spikes, consistently high peaks can be periodically seen with a uniform spatial increment, which was close to the laser pulse feed of $0.28 \mathrm{~mm} / \mathrm{pulse}$. During the experiment, the laser pulses were translated at a speed of $2.8 \mathrm{~mm} / \mathrm{sec}$ (motion stage) and a repetition rate of 10 pulses per second. The feed marks for Al and cpTi corresponded very well with the periodic high peaks in the height profiles as shown in Fig. $4 \mathrm{~b}$ and c. The height of these high peaks were about 7-9 $\mu \mathrm{m}$ and 15-18 $\mu \mathrm{m}$ for cpTi and Al, respectively. Such periodic peaks were not observed in the Cu specimens, and hence the feed marks observed for $\mathrm{Cu}$ were not due to the height profile change. 
a)

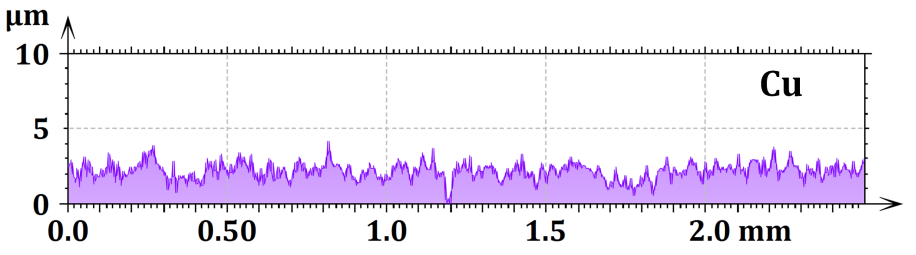

b)

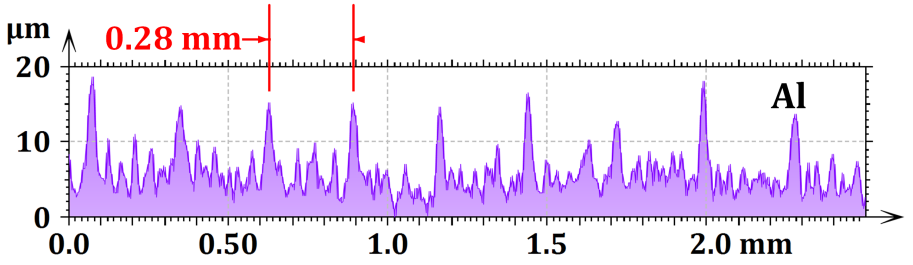

c)

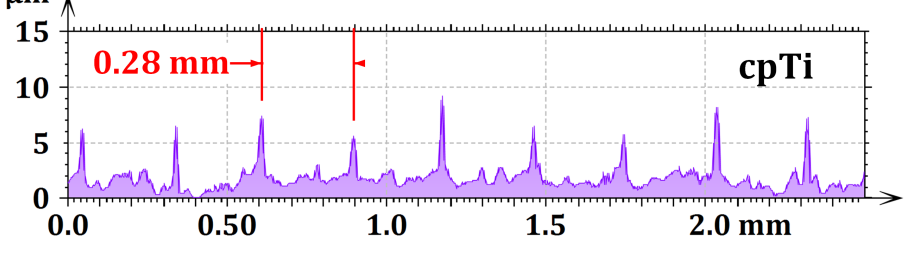

Fig. 4 Scanned surface profiles in air: a) $\mathrm{Cu}$; b) Al; c) cpTi.

To unveil how the spike-like features were formed for Al and cpTi, the local surface profile of a scanned track were compared with those of a single-shot peened surface. Fig. 5 compares the surface profile of $\mathrm{Al}$ for a) scanned tracks and b) single-shot peened spot produced using the same laser pulse in air. For a peened spot as shown in Fig. 5b, two highest peaks can be found at the two ends of the peened spot profile. The area near the high peaks of the two experiments are compared within a $200 \mu \mathrm{m}$ wide domain highlighted in the red box. For a scanned track profile, the low spikes between any two adjacent feed marks (high peaks) correspond to several small humps in the zoom-in view. These small humps observed in the scanned track were very similar to those found in a single-shot peened spot. Fig. 6 shows the SEM images near the boundary area of the single-shot peened spot for Al and cpTi. As shown in Fig. $6 \mathrm{a}$, the peened area was full of wrinkles for the $\mathrm{Al}$ specimen, which explains the many humps and peaks during the laser scanning. As can be seen in Fig. 6b, the initial rough cpTi surface was actually polished by the laser irradiation. The significant material built-up can only be seen just on the boundary of peened spot. This smooth surface within the boundary was likely formed from a calm molten pool. There were very limited tiny disordered ripples in this domain, which could be a result of Rayleigh instability of the molten pool during laser irradiation [29]. The spatial surface form was governed by the instability at the molten pool surface after the laser irradiation. In general, the instability would be resulted from the change in density during phase change and the acceleration of the liquid/solid interface. As a result, an inertial force was imposed on the molten material. This inertial force destabilized to the melt surface, while the surface tension exerted a force attempting to maintain a flat surface [30]. The above analysis 
indicates that the surface profile of a scanned track achieved in air mostly depended on the laser-induced indentation of each single shot during scanning. The feed marks of scanned tracks for $\mathrm{Al}$ and cpTi resulted from the boundary of each laser shot, and the overlapped area between two adjacent shots were modified from the next shot.
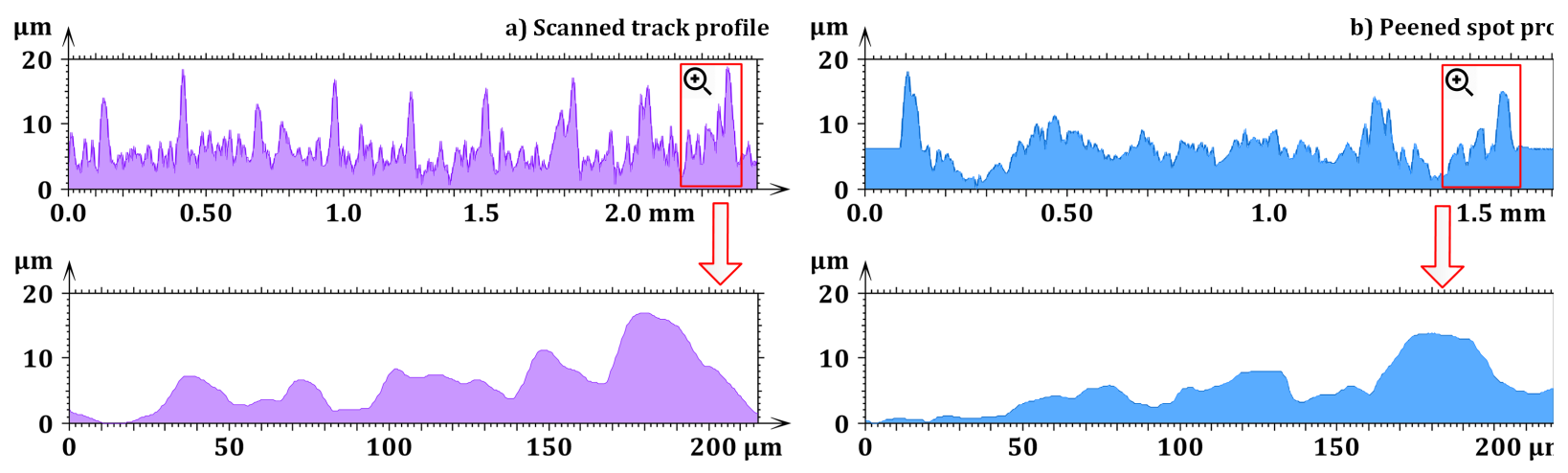

Fig. 5 Surface profile of Al produced in air: a) scanned tracks; b) single-shot peened spot.
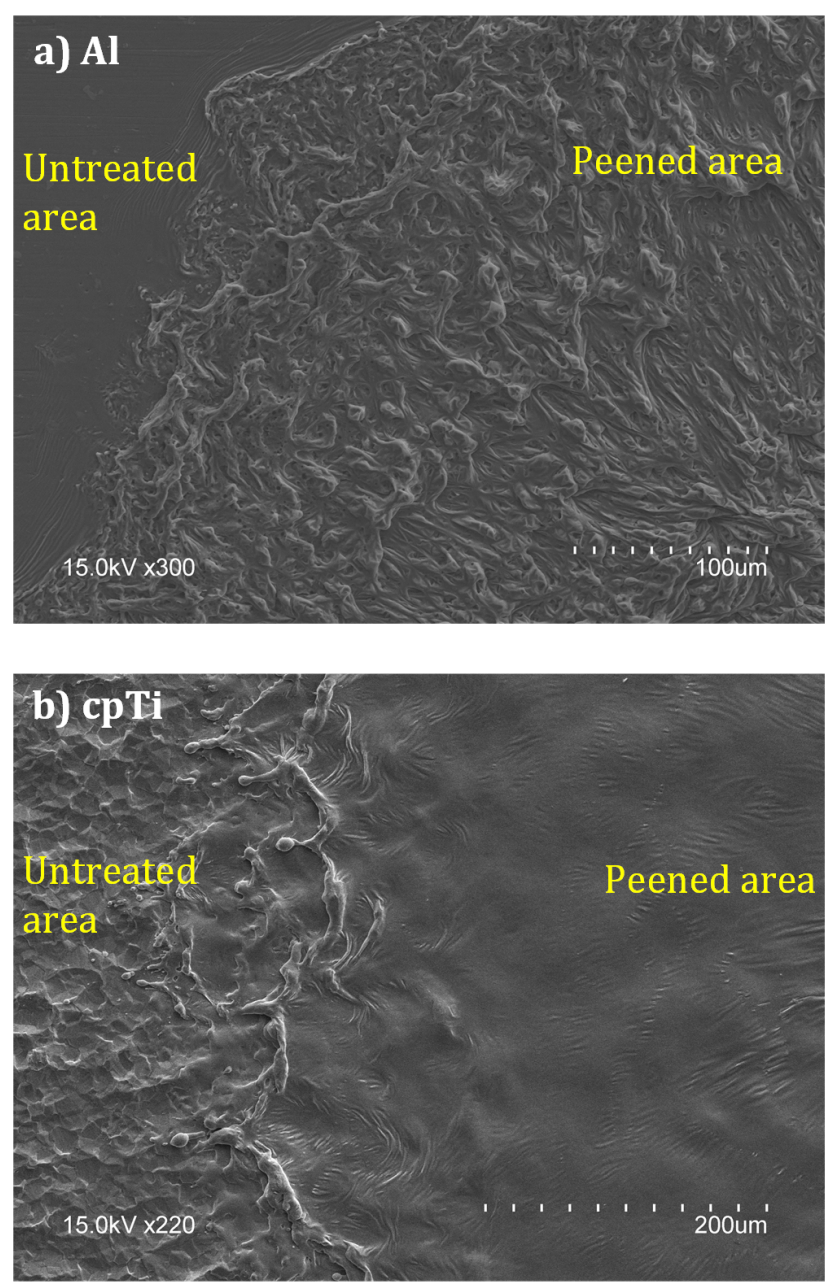

Fig. 6 SEM images near the boundary area of the peened spot with one $0.3 \mathrm{~J}$ shot in air on: a) Al; b) cpTi. 
Fig. 7 summarizes the surface roughness measurement of the three materials scanned or singleshot in air. Among the three materials, the mean roughness depth, $R_{z}$, of $\mathrm{Al}$ was the highest of about 16 $\mu \mathrm{m}(110 \%$ higher than $\mathrm{cpTi})$, while $\mathrm{Cu}$ has the lowest $R_{z}$, which corresponds well with the surface profiles as shown in Fig. 5. It is interesting to note that the scanned tracks and single-shot have very similar $R_{z}$ values, especially for $\mathrm{Al}$ and $\mathrm{cpTi}$, because the new shot formed similar high peaks from the treated area. Thus, the final surface profile of a scanned track highly depended on that of each single peened spot during the laser scanning in air. As shown in Fig. 7b, for the scanned Al specimens, the arithmetical mean roughness, $R_{a}$, increased significantly from the initial $0.08 \mu \mathrm{m}$ of the untreated area to $2.15 \mu \mathrm{m}$. Similarly for the scanned $\mathrm{Cu}$ specimens, $R_{a}$ increased from the initial $0.26 \mu \mathrm{m}$ of the untreated area to $0.45 \mu \mathrm{m}$. On the contrary, for the scanned cpTi specimens, the initial rough cpTi surface of 1.63 $\mu \mathrm{m}$ was actually polished by the laser irradiation to $0.71 \mu \mathrm{m}$. For all three materials, the $R_{a}$ values of the scanned surface was slightly higher than the single-shot spots.
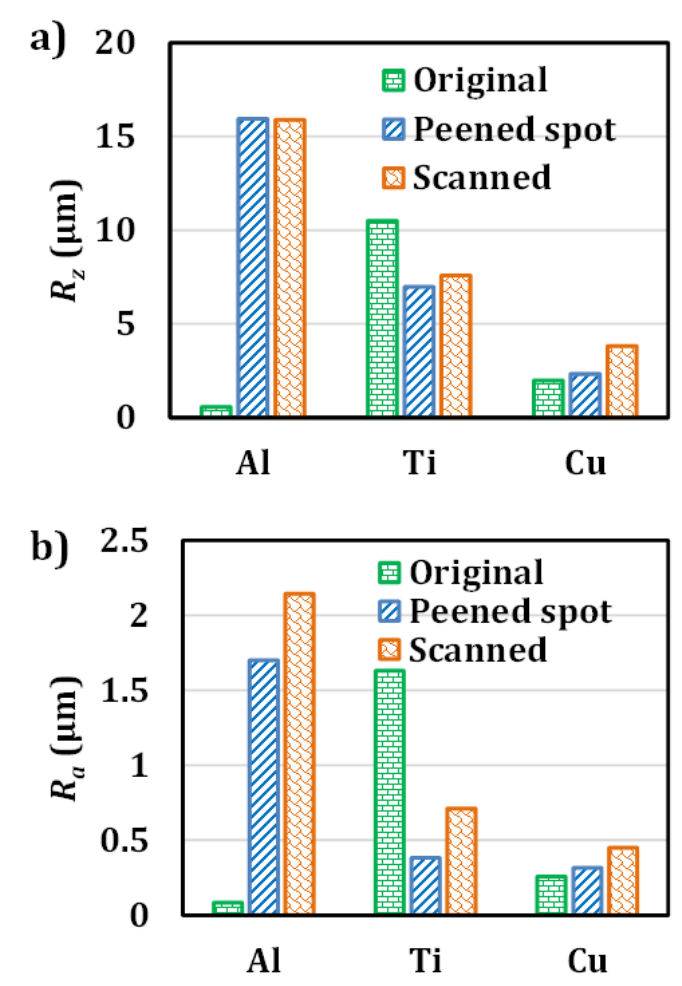

Fig. 7 Surface roughness of scanned tracks and single-shot spots produced in air: a) $R_{z} ;$ b) $R_{a}$.

Fig. 8 shows the SEM images of the top view of a Cu surface scanned by $0.4 \mathrm{~J}$ laser pulses in air. In Fig. 8a, there were six complete feed marks in dark appearance on the $\mathrm{Cu}$ surface. Fig. 8b-c show the zoom-in view of an area between two feed marks or on a feed mark. Although no significant high peaks were on the $\mathrm{Cu}$ surface, the surface between the feed marks in Fig. $8 \mathrm{~b}$ were much smoother than that on 
the feed marks in Fig. 8c. The surface texture on the feed marks looked like waves, which indicated the movement of the molten material near the boundary area of a laser spot. These surface morphology changes were too small to be detected from the $2 \mathrm{D}$ surface profile measurement using the roughness profilometer. The local surface morphological variation led to different local visual appearance in terms of color and luster. The smoother area between feed marks looked bright, but the rougher feed marks appeared dark. The above analysis showed that the surface morphology change was also the reason for the feed mark formation in OFHC $\mathrm{Cu}$.
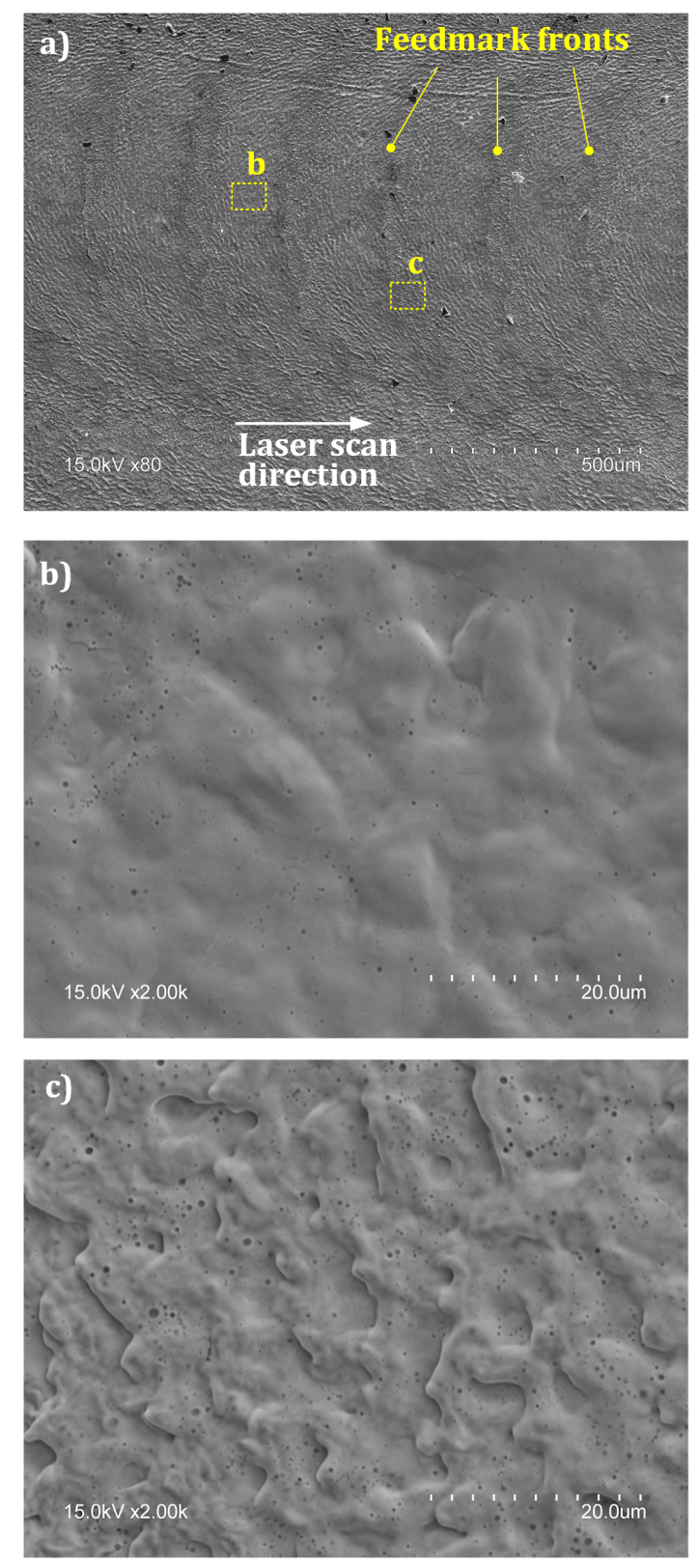

Fig. 8 Local surface on the scanned $\mathrm{Cu}$ in air: a) top view $(80 \mathrm{x})$; b) zoom-in between two feed marks $(2,000 x)$ and $c)$ on a feed mark $(2,000 x)$. 


\subsection{Surface Produced in Water}

Fig. 9 shows the surface profiles measured by the Surtronic 25 surface roughness profilometer for the specimens treated under water confinement. Different from the textures shown in Fig. 4, no periodic high peaks can be observed in the surface profiles of $\mathrm{Al}$ and cpTi. Similar to scanning in air, the $\mathrm{Cu}$ specimen has a much smoother surface than those of Al and cpTi. Fig. 10 shows the surface roughness of the scanned surface in comparison with those of single-shot spots. The scanned Al surface had the roughest profile, with both $R_{a}$ and $R_{z}$ value slightly lowered, less than $10 \%$ decrease, than those produced in air. $R_{z}$ and $R_{a}$ values for scanned cpTi surface in water were $38 \%$ and $119 \%$ higher than those produced in air, respectively. This significant surface profile difference for cpTi can be observed by comparing the profiles in water (Fig. 9c) and air (Fig. 4c). More spikes can be observed with greater heights than those in air, and the smooth surface portion between high peaks cannot be observed from Fig. 9c.

a)

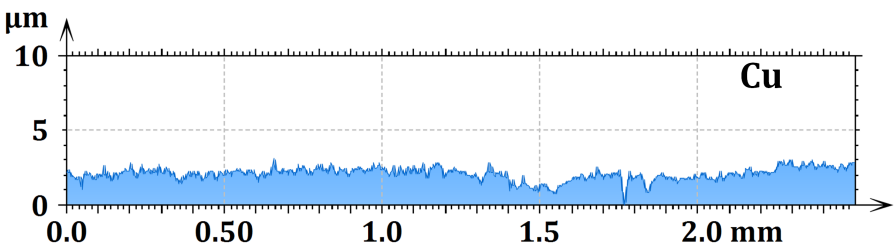

b) $\mu \mathrm{m}$

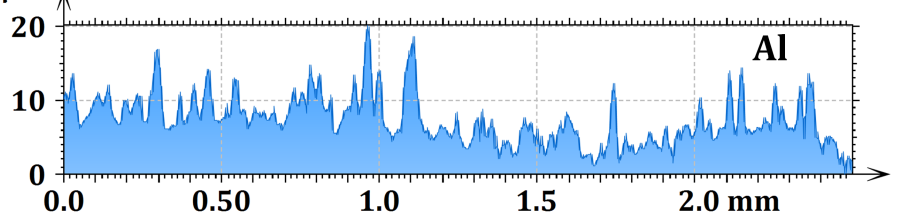

c) $\mu \mathrm{m}$

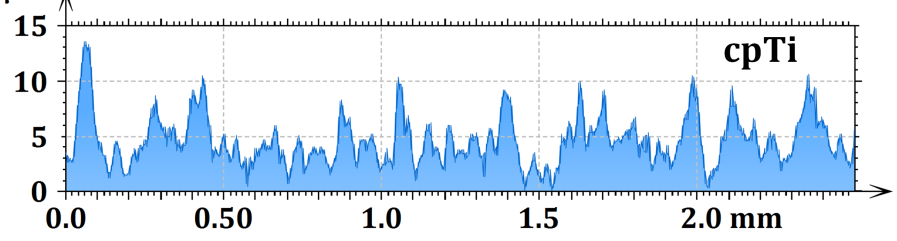

Fig. 9 Scanned surface profiles in water: a) $\mathrm{Cu}$; b) $\mathrm{Al}$; c) cpTi. 

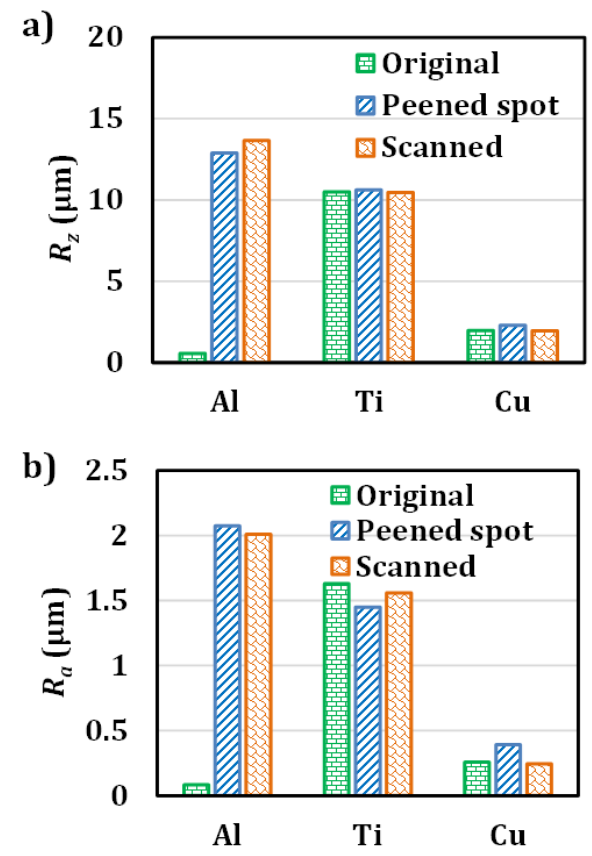

Fig. 10 Surface roughness of peened spots and scanned tracks for all three material in water: a) $\left.R_{z} ; \mathrm{b}\right) R_{a}$.

Fig. 11 shows the SEM image near the boundary area of the single-shot spot on cpTi surface in water. For the single-shot boundary area, the smooth surface in air as shown in Fig. $6 \mathrm{~b}$ was replaced with a rough surface filled with the ripples in an explosion pattern and large solidified drops. The more severe surface morphology change of cpTi in water is attributed to a more violent laser-material interaction during laser scanning. It was also found that the final surface profile of a scanned track also highly depended on that of each single peened spot during the laser scanning in water.

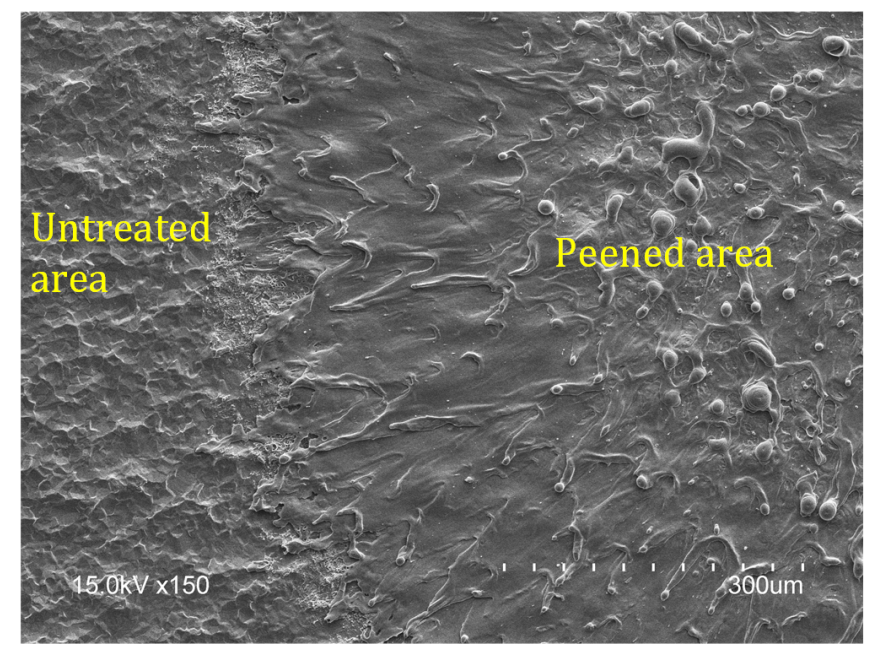

Fig. 11 SEM images near the boundary area of the peened spot on the cpTi surface with one $0.3 \mathrm{~J}$ shot (laser fluence $17.0 \mathrm{~J} / \mathrm{cm}^{2}$ ) in water. 
Although the correlation of the surface profile between a scanned track and peened spot has been found, there was not any repetive features observed on the scanned surface in water. A spatial frequency analysis (SPA) has been developed by Perry et al. [31-33] using a Fourier analysis to study the laser polished surface features and model the laser polishing process. A critical frequency, $f_{c r}$, described the cut-off point in the spatial frequency content of the surface, above which a significant reduction in the amplitude should be expected. To invesitage the LPF surface profile in spatial frequency, this SPA method was adopted in this work and a fast Fourier transformation (FFT) was performed on the laser scanned surface profile data such as those shown in Fig. 4 and 8. Fig. 12a shows the spatial frequency contents of the laser scanned surfaces for all three materials treated in air. From the spatial frequency plot, it was observed that a high spatial frequency peak was located at around $3.6 \mathrm{~mm}^{-1}$ corresponding to the feed of $0.28 \mathrm{~mm} /$ pulse and stronger harmonic frequency peaks were located at around $7.2 \mathrm{~mm}^{-1}, 10.8 \mathrm{~mm}$ ${ }^{1}$, etc. (multiples of $3.6 \mathrm{~mm}^{-1}$ ) on the laser scanned Al surface. This demonstrated that the surface features created by $0.28 \mathrm{~mm} /$ pulse feed were quite clear and distinct on the laser scanned $\mathrm{Al}$ surface in air. For the laser scanned cpTi surface, the amplitude of the spatial frequency peak at $3.6 \mathrm{~mm}^{-1}$ and its harmonics were lower than those of the Al surface. The existing spatial frequency peaks still clearly indicated the surface feature formation was attributed to the feed. However, for the laser scanned $\mathrm{Cu}$ surface in air, the amplitude of the spatial frequency peaks were much lower than those of the cpTi and Al surfaces. More spatial frequency contents and larger spatial frequency peaks will result in higher surface roughness. Thus, in air, the highest surface roughness should be expected for Al, while the lowest surface roughness should be found on $\mathrm{Cu}$ surface. These matched with the results in Fig. 7 very well, so the validity of the application of SPA was justified.

Fig. 12b shows the spatial frequency contents of the laser scanned surfaces for all three materials treated in water. Although the surface profiles of $\mathrm{Al}$ and cpTi specimens scanned in water were featureless, high spatial frequency peaks were also located at around $3.6 \mathrm{~mm}^{-1}$ and its multiples for $\mathrm{Al}$ and cpTi. Nevertheless, the amplitude of these peaks were not as significantly high as those in air as shown in Fig. 12a. Therefore, the surface profile of the scanned $\mathrm{Al}$ and cpTi surface were still related to the feed, but the random distributed surface features prevented the formation of the feed marks in water. 

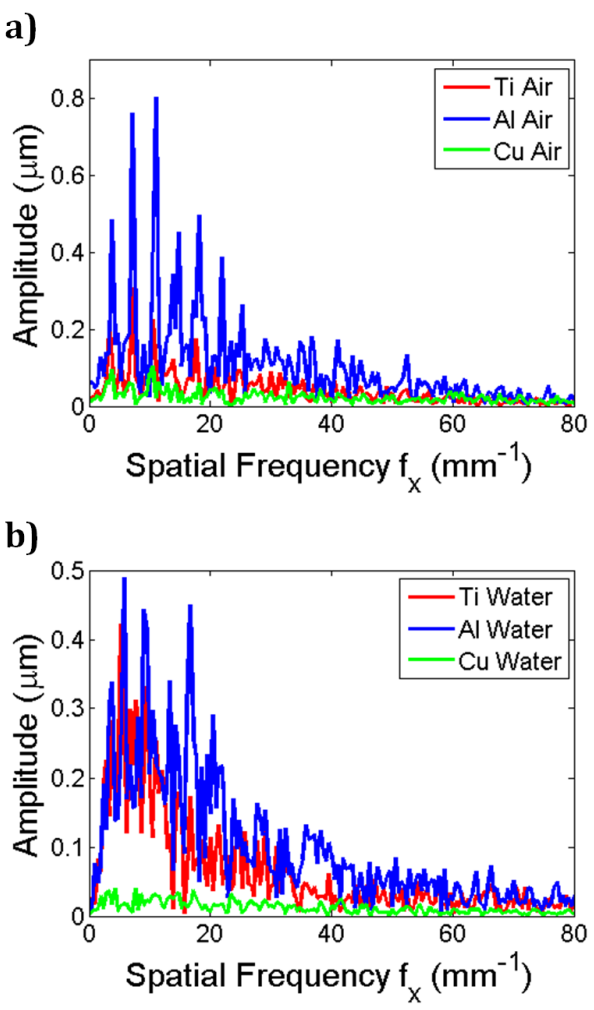

Fig. 12 Spatial frequency contents of laser scanned surface for all materials in: a) air; b) water.

The surface profile across the scanned surface in different confinement media was also investigated with the surface profilometer. Fig. 13 shows the surface profiles measured across the singlescanned tracks of $\mathrm{Al}$ and $\mathrm{Cu}$ under both confinement media. For $\mathrm{Al}$, crater-shaped surface profiles were formed with different characteristics under both confinement media. In air, the crater was mainly formed at the center with a depth less than $5 \mu \mathrm{m}$ from the untreated surface. Several high peaks were formed even above the original surface level within the track. In water, most of the scanned area was below the original surface level, which formed a much larger crater feature. The crater depth near the center roughly ranged from $5.6 \mu \mathrm{m}$ to $8.5 \mu \mathrm{m}$. The indentation under water was much more obvious than that in air. A very shallow crater of about $2.3 \mu \mathrm{m}$ was observed across the scan track on $\mathrm{Cu}$ under water confinement as shown in Fig. 13d, while the surface level was hardly changed for $\mathrm{Cu}$ under air confinement in Fig. 13b. The more significant profile change in water was attributed to a coupled effect of more violent laser ablation and greater plastic deformation under a stronger shock compression, both of which were induced by the more pronounced confinement effect of water than that of air. The results shown in Fig. 13 also indicate that material properties also played an important role on determining the surface profile change under different media. The different laser-material mechanisms are discussed the following section. 
a)

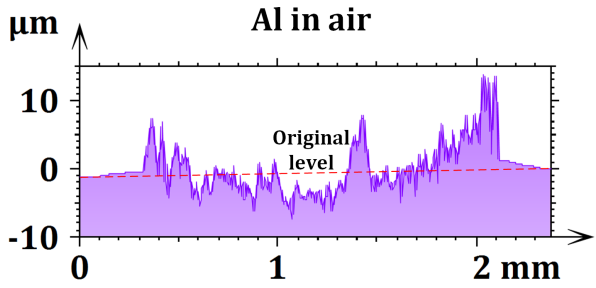

c)

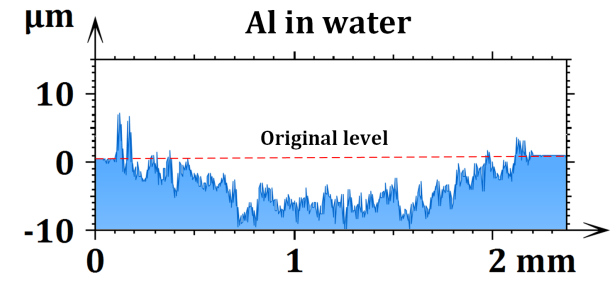

b)

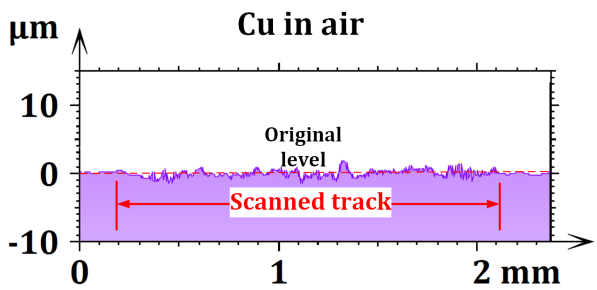

d) $\mu \mathrm{m}_{\uparrow} \quad \mathrm{Cu}$ in water

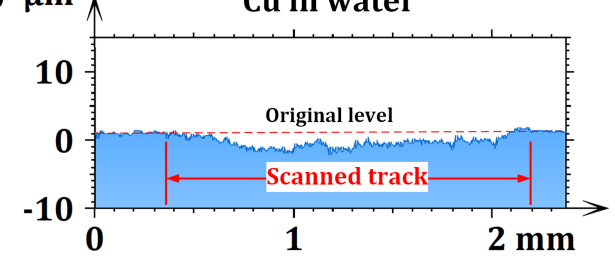

Fig. 13 Surface profile across a single scanned track for: a) $\mathrm{Al}$ in air; b) $\mathrm{Cu}$ in air; c) $\mathrm{Al}$ in water; b) $\mathrm{Cu}$ in water.

\subsection{Laser-Material Interaction Analysis}

There are two primary laser ablation mechanisms for material removal by a nanosecond laser, i.e., thermal vaporization of atoms and explosive ejection of molten droplets [29]. Within a low laser fluence range, the material would be mainly removed by the vaporization of atoms directly from the molten pool surface [34]. With the increase of laser fluence, the surface temperature of the molten pool could rise to $90 \%$ of the critical temperature $\left(T_{c}\right)$ or even higher. Under this condition, the explosive ejection of molten droplets would occur and dominate the ablation process. This phenomenon is usually referred to the explosive boiling or phase explosion [35]. The phase explosion is usually considered as the most efficient mechanism for thermal ablation on short time [36].

The transition from normal vaporization to explosive boiling has been extensively studied for metals (e.g. aluminum, platinum, and niobium), graphite, and some semiconductor materials ablated in air, water, and vacuum [29,36-43]. This transition has been explained based on the theory of metastable liquid $[29,36,37,44-47]$. During the regime of normal vaporization, the vapor plume is governed by the Hertz-Knudsen equation, in which the velocity of surface recession can be estimated [37,48]. Due to the high intensity and short duration of the laser pulse, the rapidly superheated molten material enters a metastable state which is in the region between the binodal and spinodal curves. When the molten material temperature approaches 0.8-0.9 $T_{c}$, the stability of the superheated liquid becomes drastically more sensitive on density fluctuations and the homogeneous bubble nucleation rate. The process that the superheated liquid seeks for the equilibrium would lead to its return to the binodal through phase explosion [35-38,49,50]. Based on the kinetic theory, the phase explosion would be triggered by the spontaneous expansion of bubbles when they reach a critical radius, $r_{c}$. 


\subsubsection{In Air}

Fig. 14 shows the SEM images of the zoom-in view on the scanned $\mathrm{Cu}$ surface in air. A great amount of small bubble-like pores evenly distributed on the undulating surface. Most of these pores were smaller than $0.5 \mu \mathrm{m}$ in diameter, which possibly resulted from solidification of the nucleated vapor bubbles during boiling of $\mathrm{Cu}[29]$.

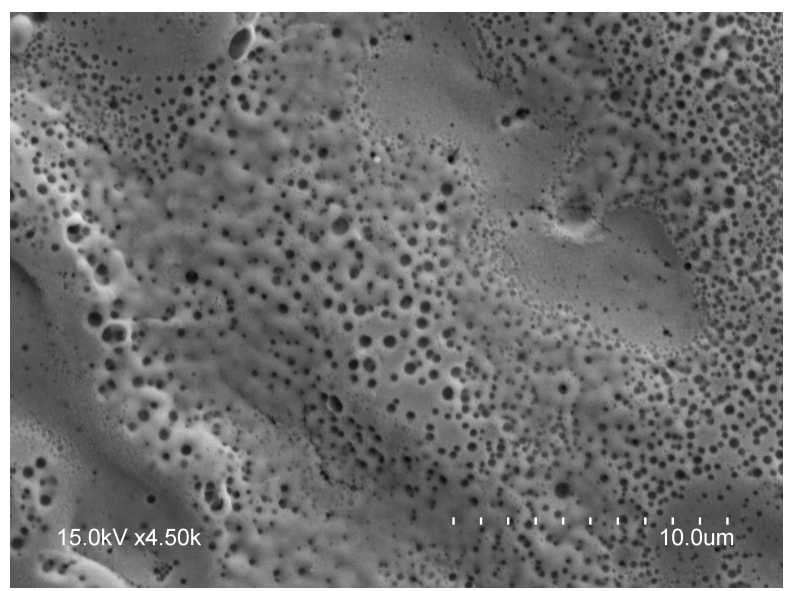

Fig. 14 Numerous bubble-like pores of on $\mathrm{Cu}$ surface scanned with $0.4 \mathrm{~J}$ pulses $\left(22.4 \mathrm{~J} / \mathrm{cm}^{2}\right)$ in air.

The critical bubble size to trigger phase explosion in laser ablation processes has been estimated based on a thermodynamic or kinetic view port $[37,38]$. The critical radius is given by Carey [51]:

$$
r_{c}=\frac{2 \sigma}{\left.P_{\text {sat }}\left(T_{1}\right) \exp \left\{v_{1} \mathbb{P}_{1}-P_{\text {sat }}\left(T_{1}\right)\right\rfloor / R T_{1}\right\} P_{1}}
$$

where $\sigma$ is the surface tension, $P_{l}$ and $T_{l}$ are the pressure and temperature of the liquid material, $P_{\text {sat }}\left(T_{l}\right)$ is saturation pressure at the liquid temperature (superheated), $v_{l}$ is the specific volume of the liquid, $R$ is the gas constant. The physical properties of $\mathrm{Al}$ and $\mathrm{Cu}$ are summarized in Table 2. $P_{l}$ was assumed to be the ambient pressure. $T_{l}$ was determined with the temperature at which the $P_{\text {sat }}$ was measured in literatures as listed in Table 2. From Eqn. 1, the estimated critical bubble radii are 1.99-2.57 $\mu \mathrm{m}$ for $\mathrm{Al}$ and 2.55-2.94 $\mu \mathrm{m}$ for $\mathrm{Cu}$. The estimated critical bubble radius of $\mathrm{Cu}$ is much greater than the bubble size observed in Fig. 14. This indicates that phase explosion was unlikely triggered during the LPF process of $\mathrm{Cu}$ in air.

Table 2. Physical properties of $\mathrm{Al}$ and $\mathrm{Cu}$.

\begin{tabular}{|c|l|l|}
\hline Properties & \multicolumn{1}{|c|}{ Al } & \multicolumn{1}{|c|}{$\mathbf{C u}$} \\
\hline Absorption (\%) & $33.7[52]$ & $7.4[52]$ \\
\hline $\boldsymbol{\rho}_{\boldsymbol{l}}\left(\mathbf{k g} / \mathbf{m}^{\mathbf{3}}\right)[53]$ & 2,375 & 8,020 \\
\hline $\boldsymbol{\rho}_{\boldsymbol{v}}\left(\mathbf{a t o m s} / \mathbf{m}^{3}\right)[54]$ & $3.22 \times 10^{24}$ for $2,790 \mathrm{~K}$ & 3.00 [010 $10^{24}$ for $2,836 \mathrm{~K}$ \\
\hline $\boldsymbol{T}_{\text {melting }}(\mathbf{K})[53]$ & 933.47 & 1357.77 \\
\hline
\end{tabular}




\begin{tabular}{|c|l|l|}
\hline $\boldsymbol{T}_{\text {boiling }}(\mathbf{K})[53]$ & 2,743 & 2,835 \\
\hline $\boldsymbol{T}_{\boldsymbol{c}}(\mathbf{K})$ & $5,115-8,944[55]$ & $8,280[56]$ \\
\hline $\boldsymbol{P}_{\text {sat }}(\mathbf{k P a})$ & 100 for 2,790 K [57] & 100 for 2,836 K [57] \\
\hline $\left.\boldsymbol{H}_{\boldsymbol{l v}} \mathbf{( M J} / \mathbf{k g}\right)[53]$ & 1076 for 3,290 K [54] & 986 for 3,337 K [54] \\
\hline & Unoxidized:1,122 for 1,276 K [58] & $4.73[53]$ \\
\hline $\boldsymbol{\sigma}(\mathbf{m N} / \mathbf{m})$ & $\begin{array}{l}\text { Oxidized: 869 for 1,276 K [58] } \\
\text { 850 for 1,373 K [59] } \\
\text { 914 above } T_{\text {melting }}[60]\end{array}$ & 1,300 for $1,357 \mathrm{~K} \mathrm{[53]}$ \\
\hline
\end{tabular}

The bubble growth rate $R(t)$, in laser ablation processes can be evaluated by [51]:

$$
R(t)=\left\{\frac{2}{3}\left[\frac{T_{1}-T_{\text {sat }}\left(P_{1}\right)}{T_{\text {sat }}\left(P_{1}\right)}\right] \frac{H_{l v} \rho_{v}}{\rho_{l}}\right\}^{1 / 2} t
$$

where $T_{\text {sat }}\left(P_{l}\right)$ is saturation temperature at the liquid pressure, i.e. boiling temperature in this case, $\rho_{v}$ and $\rho_{l}$ are the density of vapor and liquid, $H_{l v}$ is the latent heat of vaporization, all of which can be found in Table 2. The vapor density was given as atom number density in the references. From Eqn. 2, the bubble radius of $\mathrm{Al}$ can grow to $0.63 \mu \mathrm{m}$ at $20 \mathrm{~ns}$ and $3.81 \mu \mathrm{m}$ at $50 \mathrm{~ns}$, while the radius for $\mathrm{Cu}$ vapor bubble is $0.25 \mu \mathrm{m}$ at $20 \mathrm{~ns}$ and $1.51 \mu \mathrm{m}$ at $50 \mathrm{~ns}$. Therefore, the bubble in molten $\mathrm{Al}$ can reach the critical bubble radius even earlier, because it is expected to grow much faster than that in molten $\mathrm{Cu}$.

The critical temperature of $\mathrm{Al}$ has been reported to have a wide range from $5,115 \mathrm{~K}$ to $8,944 \mathrm{~K}$ [55], while that of $\mathrm{Cu}$ was reported as $8,280 \mathrm{~K}$ [56]. Cao and Shin [50] recently conducted a multi-scale modeling work on high energy nanosecond laser ablation and predicted the occurrence of phase explosion and the corresponding laser ablation depth. The critical temperature of $\mathrm{Al}$ was predicted from molecular dynamic simulations and was narrowed to the range of 5,950-6,200 K, about 2,000 K lower than $\mathrm{Cu}$. Furthermore, $\mathrm{Cu}$ has a much lower absorption to laser with wavelength of $1064 \mathrm{~nm}$ than $\mathrm{Al}$. The low absorption makes it is even more difficult to satisfy the condition to trigger phase explosion for $\mathrm{Cu}$ in air.

For Nd:YAG laser (wavelength of $1064 \mathrm{~nm}$ ), it has been reported that the transition from vaporization to phase explosion would occur at about $7.5 \mathrm{~J} / \mathrm{cm}^{2}$ with a $100 \mu \mathrm{m}$ laser spot size for Al in air [63], while the phase explosion would occur when the laser fluence is greater than about $30 \mathrm{~J} / \mathrm{cm}^{2}$ with a $100-250 \mu \mathrm{m}$ spot size for $\mathrm{Cu}$ in air [64,65]. In this study, the laser fluence varied from 17 to $22.4 \mathrm{~J} / \mathrm{cm}^{2}$ with the laser pulse energy from 0.3 to $0.4 \mathrm{~J}$. Thus, the phase explosion was considered to have happened for $\mathrm{Al}$ specimens treated in air. On the other hand, phase explosion was unlikely to have occurred for $\mathrm{Cu}$ specimens treated in air, since the maximum laser fluence of $22.4 \mathrm{~J} / \mathrm{cm}^{2}$ used in this study was less than the reported value of $30 \mathrm{~J} / \mathrm{cm}^{2}$. The aforementioned analyses explain the significant surface profile differences of $\mathrm{Al}$ and $\mathrm{Cu}$ as shown in Fig. 4 and the surface depth change illustrated in Fig. 13. 


\subsubsection{In Water}

It has been reported that the transition from vaporization to phase explosion for $\mathrm{Al}$ occurs under about $5.7 \mathrm{~J} / \mathrm{cm}^{2}$ in water for the $1064 \mathrm{~nm} \mathrm{Nd}$ :YAG laser with a 1,000 $\mu \mathrm{m}$ spot size [41]. This laser fluence for $\mathrm{Al}$ specimen treated in water was lower than those in air, even with a much bigger laser spot size. This is due to the dense confinement of water, which reduces heat loss [49]. In air, a strong ionized vapor plume is ejected out of the surface, carrying away much hot target material. In water, the expansion of the vapor plume is significantly confined as the stronger confinement effect. The drastic decrease of the ejected hot mass would reduce the heat loss from evaporative cooling of the target, resulting in higher temperatures. In addition, void formation would be boosted with the more severe thermal expansion in the high temperature target surface layer during the nanosecond laser irradiation. More bubbles in the molten material reduce the thermal conductance, so it would be more difficult for heat to transfer into the bulk material [66]. Therefore, phase explosion was determined to have happened for $\mathrm{Al}$ in water under the laser fluence of $17-22.4 \mathrm{~J} / \mathrm{cm}^{2}$ in this work.

The transition laser fluence of $\mathrm{Cu}$ in water is expected to be lower than $30 \mathrm{~J} / \mathrm{cm}^{2}$ in air. However, due to lack of experimental data of $\mathrm{Cu}$, the occurrence of the phase explosion in water cannot be determined directly by comparing the laser fluence to a transition threshold. Nonetheless, the phase explosion was considered not to have occurred. Fig. 15a shows a raster-scanned area of $\mathrm{Cu}$ in water in SEM. A uniform and featureless surface morphology can be seen all over the scanned area. When examining the scanned area with more details in Fig. 15b, irregular small ripples were uniformly distributed all over the surface. The bubble-like pores cannot be seen under this condition. These observations for $\mathrm{Cu}$ were completely different from the peened $\mathrm{Al}$ surface with many explosion-like features and droplets after phase explosion as shown in Fig. 15c. From the above analyses, phase explosion was considered to have happened for $\mathrm{Al}$ under all the conditions in both air and water, but have not occurred for $\mathrm{Cu}$ under all the conditions.
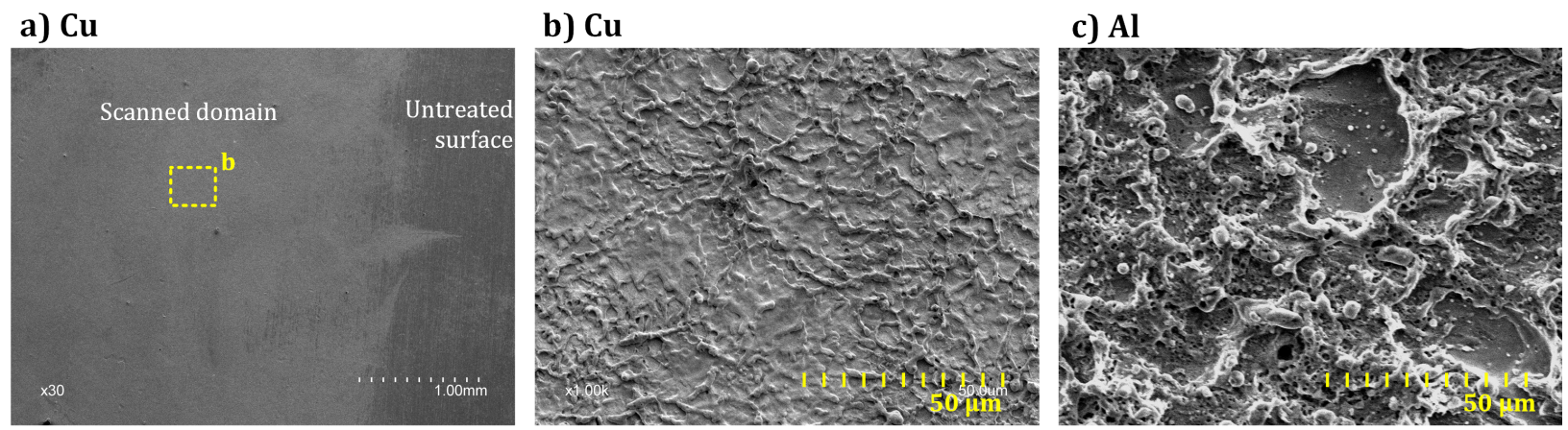

Fig. $15 \mathrm{SEM}$ images of scanned/peened surfaces with $0.4 \mathrm{~J}$ pules (laser fluence $22.4 \mathrm{~J} / \mathrm{cm}^{2}$ ) in water: a) scanned $\mathrm{Cu}(30 \mathrm{x})$; b) scanned $\mathrm{Cu}(1,000 \mathrm{x})$; c) single-shot peened spot of $\mathrm{Al}(1,000 \mathrm{x})$. 
Based on the morphology of single-shot peened cpTi surface as shown in Fig. 16, phase explosion was suggested to have occurred for cpTi in water, but not in air. As shown in Fig. 16a, the surface was actually polished by the laser irradiation in air, and seemed like a solidified calm molten pool. In contrast, an obvious explosion pattern can be seen from the surface treated in water as shown in Fig. 16b. It seemed that the molten pool quickly solidified as the material was being ejected from the center. Multiple waves of molten material can be easily seen, which also indicated a more violent laser-material interaction in water for cpTi. The occurrence of phase explosion in water induced a rougher surface morphology on cpTi specimen that that treated in air with no phase explosion.
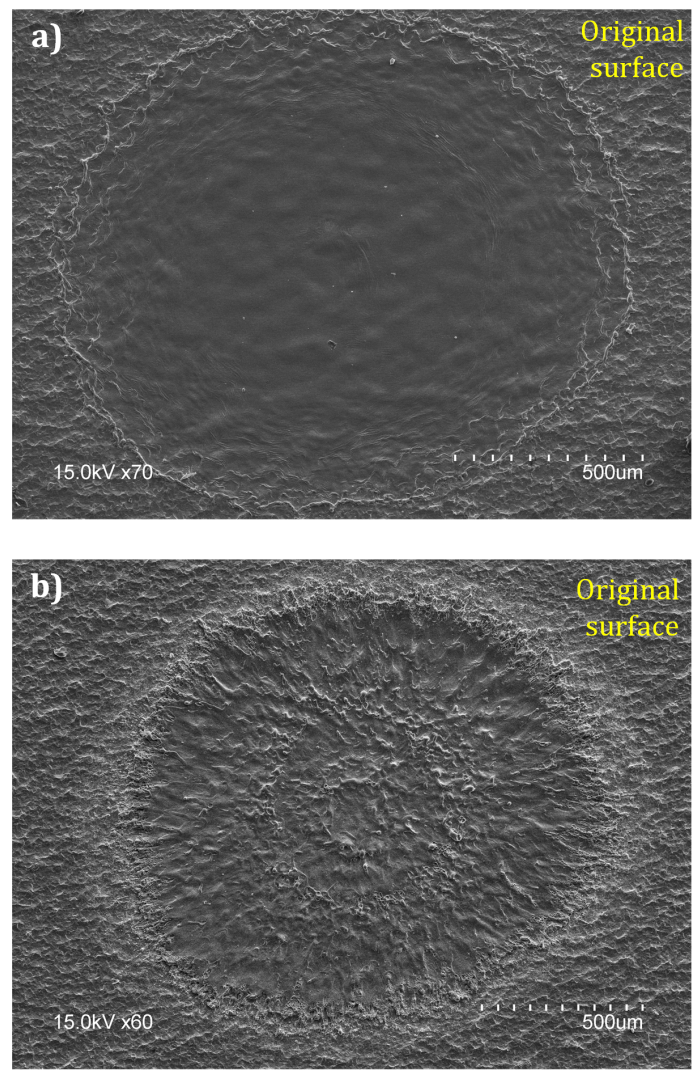

Fig. 16 SEM images of single-shot peened cpTi surface with $0.4 \mathrm{~J}$ pule (laser fluence $22.4 \mathrm{~J} / \mathrm{cm}^{2}$ ) in: a) air; b) water.

\section{Chemical Analysis}

A chemical composition analysis was performed on the scanned surfaces by a surface-sensitive quantitative spectroscopic technique of X-ray photoelectron spectroscopy (XPS) using a high-sensitivity 
Kratos ${ }^{\circledR}$ Axis Ultra XPS System. The chemical composition change on the $\mathrm{Cu}$ surface scanned with $0.4 \mathrm{~J}$ pulses under water or air confinement was investigated in this study. The relative intensity of different energy levels was characterized as count per second (CPS) for the photoelectrons at any specific energy level in this study. The measured data were processed and visualized using a software package of CasaXPS. Before the identification of each peak, the whole recorded wide-scan spectrum was aligned with the binding energy of $\mathrm{C} 1 \mathrm{~s}$. The depth profiling function was obtained by combining a sequence of $\mathrm{Ar}^{+}$ion gun etch cycles before next XPS measurement on the new surface. Thus, the chemical composition change in depth was also investigated.

Fig. 17a shows the wide scan (dwell time $200 \mathrm{~ms}$ and energy resolution of $0.5 \mathrm{eV}$ ) results through the whole energy spectrum for a polished original $\mathrm{Cu}$ surface. The significant two high peaks (about $14.4 \times 10^{5} \mathrm{CPS}$ and $21.2 \times 10^{5} \mathrm{CPS}$ ) on the left of the figure correspond to the spin orbit doublet of $\mathrm{Cu} 2 \mathrm{p}_{1 / 2}$ and $\mathrm{Cu} 2 \mathrm{p}_{3 / 2}$ with the binding energy of $952.8 \mathrm{eV}$ and $933 \mathrm{eV}$, respectively. There was also a spike for $\mathrm{Cu}$ $3 p$ with the binding energy of $74 \mathrm{eV}$ close to the right end of the spectrum. Furthermore, the rest peaks did not have a comparable intensity. The mass and atomic concentration of $\mathrm{Cu}$ were very high on this surface, which were $99.50 \%$ and $98.05 \%$, respectively. After the laser scanning with $0.4 \mathrm{~J}$ pulses in water, the $\mathrm{Cu}$ surface wide-scan energy spectrum was significantly changed as shown in Fig. 17b. The two high peaks of spin orbit doublet of $\mathrm{Cu}$ dropped to $4.70 \times 10^{5} \mathrm{CPS}$ (by 67.36\%) and $5.39 \times 10^{5} \mathrm{CPS}$ (by $74.58 \%$ ), respectively. The intensity of peaks for $\mathrm{O} 1 \mathrm{~s}$ and $\mathrm{C} 1 \mathrm{~s}$ were about $1.37 \times 10^{5} \mathrm{CPS}$ and $1.10 \times 10^{5} \mathrm{CPS}$, respectively, much higher than those for an original $\mathrm{Cu}$ surface in Fig. 17a. Moreover, there was even a tiny spike for $\mathrm{N} 1 \mathrm{~s}$ with intensity about $4.68 \times 10^{4} \mathrm{CPS}$. These change was likely to be attributed to the oxidization and organic containments in water. As shown in Fig. 17c, by etching the scanned surface by $30 \mathrm{~nm}$ using the ion gun, the wide-scan energy spectrum almost restored to the same intensity of spectra of an original $\mathrm{Cu}$ surface as shown in Fig. 17a. Thus, the chemical composition change has vanished at the depth of $30 \mathrm{~nm}$.
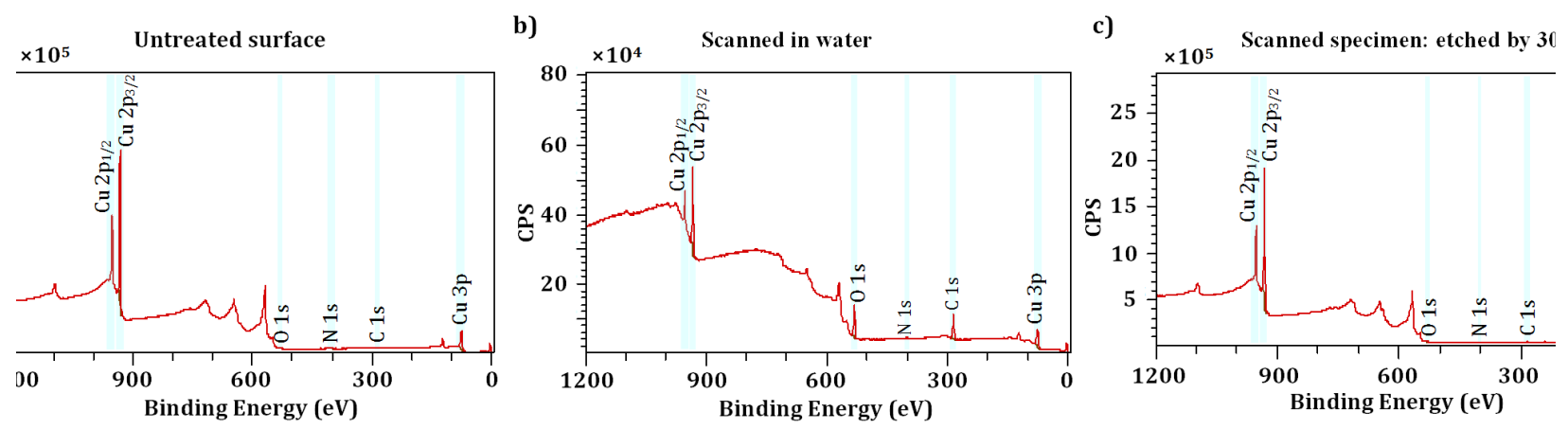

Fig. 17 Full spectrum survey for $\mathrm{Cu}$ specimen. a) Untreated polished surface; b) Scanned specimen in water; c) Scanned specimen etched by $30 \mathrm{~nm}$ in depth. 
To investigate the surface chemical composition change along depth in details, depth profiling was carried out for $\mathrm{Cu} 2 \mathrm{p}$ and $\mathrm{O} 1 \mathrm{~s}$ with higher energy resolution of $0.1 \mathrm{eV}$ on the scanned $\mathrm{Cu}$ surface under water or air confinement. The surface was etched by $2 \mathrm{~nm}$ by 10 ion-gun etching cycles between two adjacent XPS measurements. Fig. 18a-b show the depth profile of $\mathrm{Cu} 2 \mathrm{~s}$ and $\mathrm{O} 1 \mathrm{~s}$ with the depth incremental of $2 \mathrm{~nm}$. As shown in Fig. 18a, there was a drastic increase of the intensity of $\mathrm{Cu} 2 \mathrm{p}$ just on the $2 \mathrm{~nm}$ deep surface, and it reached a steady-state intensity before $10 \mathrm{~nm}$ deep. For $\mathrm{O} 1 \mathrm{~s}$, due to the higher resolution, an additional shifted peak for $\mathrm{O} 1 \mathrm{~s}$ was captured at the binding energy of $531.56 \mathrm{eV}$ from the peak of $530 \mathrm{eV}$ on the as-peened $\mathrm{Cu}$ surface. For the two specific binding energies of $531.56 \mathrm{eV}$ and $530 \mathrm{eV}$ correspond the chemical state of organic C-O bond and metal oxides. However, this shifted peak vanished at $2 \mathrm{~nm}$ deep surface. The peak of $530 \mathrm{eV}$ was the only one left for $\mathrm{O} 1 \mathrm{~s}$ on the $2 \mathrm{~nm}$ deep surface and also the following XPS measurements at deeper surfaces. In addition, the intensity of O $1 \mathrm{~s}$ significantly decreased until a steady-state intensity reached at depth about 8-10 nm. Thus, the shifted O $1 \mathrm{~s}$ peak on the as-peened surface was attributed to the surface hydration and organic contaminants in water, and all the oxygen existed only as the oxides of copper below the as-peened surface. Fig. 18c shows the depth profiles of mass and atomic concentrations for $\mathrm{Cu}$ and oxygen of the scanned $\mathrm{Cu}$ surface. On the scanned surface, $\mathrm{Cu}$ started with very low mass and atomic concentration of $69.81 \%$ and $36.80 \%$, respectively, while those of oxygen were $30.19 \%$ and $63.20 \%$. Similar to the intensity change of $\mathrm{Cu} 2 \mathrm{p}$ and $\mathrm{O} 1 \mathrm{~s}$, at the depth of $2 \mathrm{~nm}$, the mass and atomic concentration of $\mathrm{Cu}$ greatly increased to $96.16 \%$ and $86.31 \%$, respectively, while those of oxygen dropped to $3.84 \%$ and $13.69 \%$. At the depth of $10 \mathrm{~nm}$, the mass and atomic concentration of $\mathrm{Cu}$ were $98.62 \%$ and $94.74 \%$, both of which have reached a level close to those of OFHC copper ( $99.50 \%$ and $98.05 \%$ ). Both mass and atomic concentration of $\mathrm{Cu}$ have been already restored to the level of OFHC copper at depth of $30 \mathrm{~nm}$. Thus, the chemical composition change and chemical contamination on the scanned $\mathrm{Cu}$ surface under water confinement were limited within the depth of $30 \mathrm{~nm}$. 
pth profile

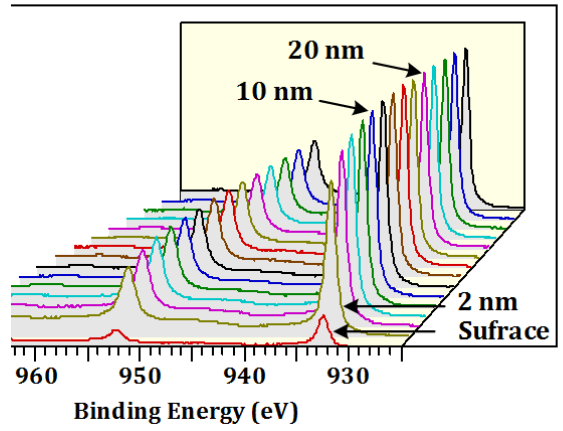

b) $01 \mathrm{~s}$ depth profile

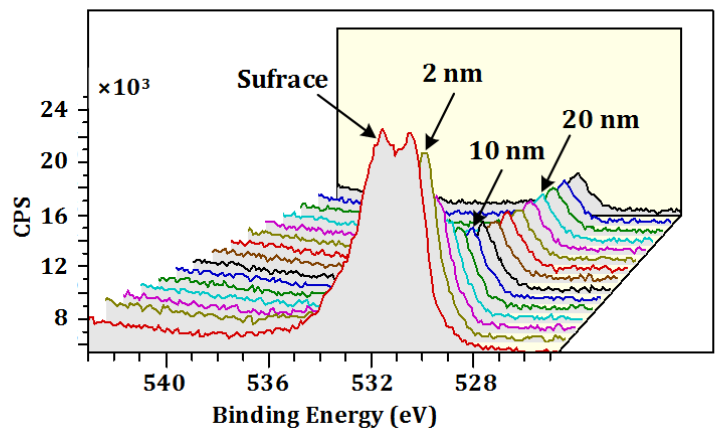

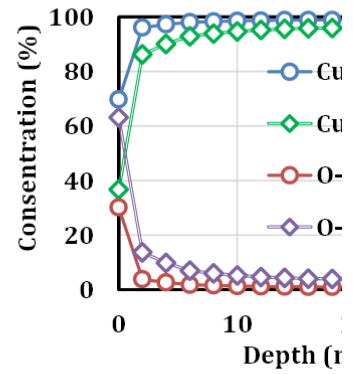

Fig. 18 Chemical analysis on the depth profiles for $\mathrm{Cu}$ surface scanned with $0.4 \mathrm{~J}$ laser pulses in water: a) $\mathrm{CPS}$ for $\mathrm{Cu} 2 \mathrm{p}$; b) $\mathrm{O} 1 \mathrm{~s}$; c) mass/atomic concentrations of $\mathrm{Cu} 2 \mathrm{p}$ and $\mathrm{O} 1 \mathrm{~s}$.

Similar XPS results were observed on the $\mathrm{Cu}$ surface scanned in air, but the surface composition change was more significant than that under water confinement. Fig. 19 shows the comparison of $\mathrm{Cu}$ and $\mathrm{O}$ atomic concentration change in depth for $\mathrm{Cu}$ surface scanned with $0.4 \mathrm{~J}$ laser pulses under water or air confinement. The atomic concentration of $\mathrm{Cu}$ and $\mathrm{O}$ on treated surface in air were $12.36 \%$ and $87.64 \%$, respectively, which were $36.8 \%$ and $63.2 \%$ in water. Significantly more oxidization in air should contribute most of the increase of the atomic concentration of $\mathrm{O}$ on the surface. However, these chemical composition change also diminished within $30 \mathrm{~nm}$ in depth for the surface treated under air.

a) $\mathrm{Cu}$

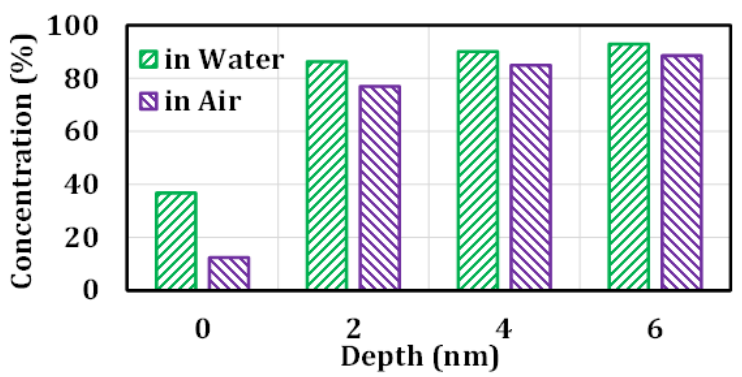

b) 0

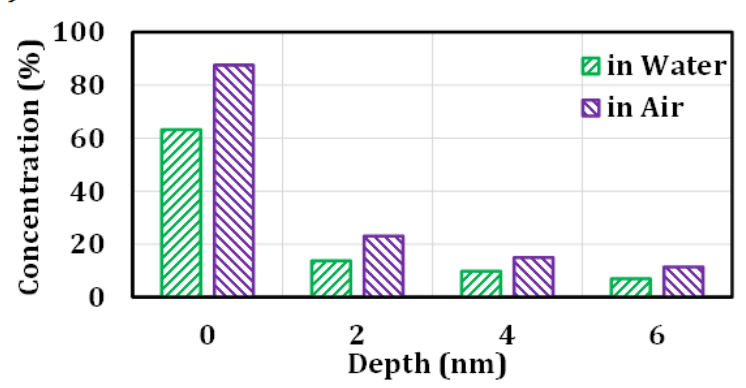

Fig. 19 Comparisons of $\mathrm{Cu}$ and $\mathrm{O}$ atomic concentration change in depth for $\mathrm{Cu}$ surface scanned with 0.4J laser pulses in both water and air: a) $\mathrm{Cu}$; b) $\mathrm{O}$. 


\section{Conclusions}

The effects of confinement on surface morphological and composition changes by LPF was studied through laser peen micro-bending experiments. Clear feed mark patterns were observed corresponding to the laser pulse feed of $0.28 \mathrm{~mm} /$ pulse on the scanned surface for all three materials under air confinement. For the materials treatment under water confinement, the specimen surface appeared in a tarnished, featureless surface texture without showing any feed marks. The feed mark formation under air confinement for all three materials was determined due to the surface morphology change by LPF. Surface profile analysis showed that the feed marks for Al and cpTi were due to the periodic high peaks, while SEM analysis showed that local morphological variations led to the feed mark formation for $\mathrm{Cu}$. XPS analysis showed surface chemical composition changes due to the hightemperature and high-pressure process condition during LPF. The surface chemical composition change was limited within the topmost 10-30 nm from the scanned surface under both water and air confinements. The surface microhardness was found to increase by $38 \%$ to $83 \%$ for different materials. Compressive residual stress at least -207 MPa was induced on the treated surface.

The surface morphology resulted from the mechanism of laser-material interaction under different medium confinement. Phase explosion was determined to have happened for Al under both air and water confinement, which resulted in a remarkable increase in surface roughness by over 20 -fold. It absented during scanning of $\mathrm{Cu}$ specimens in both media due to its high critical temperature, low laser energy absorption, large critical bubble size, etc. Phase explosion was determined to have occurred for

cpTi under water confinement but not in air. In air, the initial rough cpTi surface was actually polished by the laser irradiation without phase explosion. The water medium confines the ejected hot mass during laser scanning, leads to a more violent laser-material interaction and higher possibility of phase explosion. Due to phase explosion, surface roughness for scanned cpTi surface in water increased significantly from those produced in air.

\section{Acknowledgments}

Discussions of surface morphology with Dr. Benxin $\mathrm{Wu}$ from Purdue University are gratefully appreciated. The authors gratefully acknowledge the financial support provided for the part of the study carried out at the University of Iowa by the National Science Foundation under Grant Number EPS1101284 and State Key Laboratory of Mechanical System and Vibration, Shanghai Jiao Tong University, China under Grant Number MSV201514. 


\section{References}

[1] K.R. Edwards, S.P. Edwardson, C. Carey, G. Dearden, K.G. Watkins, Laser micro peen forming without a tamping layer, The International Journal of Advanced Manufacturing Technology. 47 (2009) 191-200.

[2] Y. Sagisaka, M. Kamiya, M. Matsuda, Y. Ohta, Thin-sheet-metal bending by laser peen forming with femtosecond laser, Journal of Materials Processing Technology. 210 (2010) 2304-2309. doi:http://dx.doi.org/10.1016/j.jmatprotec.2010.08.025.

[3] B. Wu, Y.C. Shin, From incident laser pulse to residual stress: a complete and self-closed model for laser shock peening, Journal of Manufacturing Science and Engineering. 129 (2007) 117-125.

[4] H. Ding, Y.C. Shin, Dislocation density-based modeling of subsurface grain refinement with laser-induced shock compression, Computational Materials Science. 53 (2012) 79-88. doi:10.1016/j.commatsci.2011.08.038.

[5] N. Shen, H. Ding, R. Bowers, Y. Yu, C.N. Pence, I.T. Ozbolat, et al., Surface Micropatterning of Pure Titanium for Biomedical Applications Via High Energy Pulse Laser Peening, Transactions of the ASME, Journal of Micro and Nano-Manufacturing. 3 (2015) 11005. doi:10.1115/1.4029247.

[6] Y.X. Hu, X.X. Xu, Z.Q. Yao, J. Hu, Laser peen forming induced two way bending of thin sheet metals and its mechanisms, Journal of Applied Physics. 108 (2010). doi:Artn 073117Doi 10.1063/1.3486218.

[7] Y. Guan, H. Zhang, J. Liu, S. Sun, Laser micro-bending process based on the characteristic of the laser polarization, Journal of Materials Processing Technology. 212 (2012) 662-671. doi:10.1016/j.jmatprotec.2011.07.010.

[8] J. Ocana, M. Morales, C. Molpeceres, O. Garcia, J. Porro, J. Garciaballesteros, Short pulse laser microforming of thin metal sheets for MEMS manufacturing, Applied Surface Science. 254 (2007) 9971001.

[9] J.L. Ocaña, Nanosecond Laser Shock Microforming of Thin Metal Components, Journal of Laser Micro/Nanoengineering. 4 (2009) 55-60.

[10] X.R. Zhang, X. Xu, Laser bending for adjusting curvatures of hard disk suspensions, Microsystem Technologies. 11 (2005) 1197-1203. doi:10.1007/s00542-005-0588-3.

[11] X.R. Zhang, X. Xu, Laser bending for high-precision curvature adjustment of microcantilevers, Applied Physics Letters. 86 (2005) 021114. doi:10.1063/1.1851617.

[12] M. Geiger, F. Meyer-Pittroff, Laser beam bending of metallic foils, in: I. Miyamoto, Y.F. Lu, K. Sugioka, J.J. Dubowski (Eds.), Second International Symposium on Laser Precision Micromachining, International Society for Optics and Photonics, 2002: pp. 187-190. doi:10.1117/12.456823.

[13] L. Hackel, F. Harris, Contour forming of metals by laser peening, 2002. http://www.google.com/patents/US6410884.

[14] A. Vasu, R. V. Grandhi, Effects of curved geometry on residual stress in laser peening, Surface and Coatings $\quad$ Technology. 218 (2013) 71-79. http://www.sciencedirect.com/science/article/pii/S0257897212012753 (accessed January 22, 2016).

[15] A. Vasu, Y. Hu, R. V. Grandhi, Differences in plasticity due to curvature in laser peened components, $\begin{array}{lllll}\text { Surface } \quad \text { and } & \text { Coatings } & \text { Technology. }\end{array}$ http://www.sciencedirect.com/science/article/pii/S0257897213008207 (accessed January 22, 2016).

[16] H. Ding, N. Shen, K. Li, W. Bo, C.N. Pence, H. Ding, Experimental and Numerical Analysis of Laser Peen Forming Mechanisms of Sheet Metal, in: Proceedings of ASME 2014 International Manufacturing Science and Engineering Conference, MSEC2014-4210, Detroit, Michigan, USA, 2014. doi:10.1115/MSEC20144210.

[17] C. Pence, H. Ding, N. Shen, H. Ding, Experimental analysis of sheet metal micro-bending using a nanosecond-pulsed laser, The International Journal of Advanced Manufacturing Technology. 69 (2013) 319-327. doi:10.1007/s00170-013-5032-8. 
[18] G. Dearden, S.P. Edwardson, Some recent developments in two-and three-dimensional laser forming for macro and micro applications, Journal of Optics A: Pure and Applied Optics. 5 (2003) S8-S15. doi:10.1088/1464-4258/5/4/352.

[19] U. Trdan, M. Skarba, J. Grum, Laser shock peening effect on the dislocation transitions and grain refinement of Al-Mg-Si alloy, Materials Characterization. 97 (2014) 57-68. doi:10.1016/j.matchar.2014.08.020.

[20] Y. Wang, Y. Fan, S. Vukelic, Y. Lawrence Yao, Energy-Level Effects on the Deformation Mechanism in Microscale Laser Peen Forming, Journal of Manufacturing Processes. 9 (2007) 1-12. doi:10.1016/S15266125(07)70103-2.

[21] C. Ye, G.J. Cheng, Effects of Temperature on Laser Shock Induced Plastic Deformation: The Case of Copper, Journal of Manufacturing Science and Engineering. 132 (2010) 061009. doi:10.1115/1.4002849.

[22] Y. Shadangi, K. Chattopadhyay, S.B. Rai, V. Singh, Effect of LASER shock peening on microstructure, mechanical properties and corrosion behavior of interstitial free steel, Surface and Coatings Technology. 280 (2015) 216-224. doi:10.1016/j.surfcoat.2015.09.014.

[23] U. Trdan, J. Grum, Evaluation of corrosion resistance of AA6082-T651 aluminium alloy after laser shock peening by means of cyclic polarisation and ElS methods, Corrosion Science. 59 (2012) 324-333. doi:10.1016/j.corsci.2012.03.019.

[24] U. Trdan, J. Grum, SEM/EDS characterization of laser shock peening effect on localized corrosion of Al alloy in a near natural chloride environment, Corrosion Science. 82 (2014) 328-338. doi:10.1016/j.corsci.2014.01.032.

[25] C. Gu, Z. Shen, H. Liu, P. Li, M. Lu, Q. Zhang, et al., Investigation on bend displacement and surface quality induced by laser shock micro-adjustment, Applied Surface Science. 270 (2013) 281-286. doi:10.1016/j.apsusc.2012.12.174.

[26] S. Kalainathan, S. Sathyajith, S. Swaroop, Effect of laser shot peening without coating on the surface properties and corrosion behavior of 316L steel, Optics and Lasers in Engineering. 50 (2012) 1740-1745. doi:10.1016/j.optlaseng.2012.07.007.

[27] D. Karthik, S. Kalainathan, S. Swaroop, Surface modification of 17-4 PH stainless steel by laser peening without protective coating process, Surface and Coatings Technology. 278 (2015) 138-145. doi:10.1016/j.surfcoat.2015.08.012.

[28] U. Trdan, J.A. Porro, J.L. Ocaña, J. Grum, Laser shock peening without absorbent coating (LSPwC) effect on 3D surface topography and mechanical properties of 6082-T651 Al alloy, Surface and Coatings Technology. 208 (2012) 109-116. doi:10.1016/j.surfcoat.2012.08.048.

[29] W.T. Nichols, T. Sasaki, N. Koshizaki, Laser ablation of a platinum target in water. I. Ablation mechanisms, Journal of Applied Physics. 100 (2006) 114911. doi:10.1063/1.2390640.

[30] T.D. Bennett, C.P. Grigoropoulos, D.J. Krajnovich, Near-threshold laser sputtering of gold, Journal of Applied Physics. 77 (1995) 849. doi:10.1063/1.359010.

[31] T.L. Perry, D. Werschmoeller, N.A. Duffie, X. Li, F.E. Pfefferkorn, Examination of Selective Pulsed Laser Micropolishing on Microfabricated Nickel Samples Using Spatial Frequency Analysis, Journal of Manufacturing Science and Engineering. 131 (2009) 021002. doi:10.1115/1.3075874.

[32] T.L. Perry, D. Werschmoeller, X. Li, F.E. Pfefferkorn, N.A. Duffie, The Effect of Laser Pulse Duration and Feed Rate on Pulsed Laser Polishing of Microfabricated Nickel Samples, Journal of Manufacturing Science and Engineering. 131 (2009) 031002. doi:10.1115/1.3106033.

[33] T.L. Perry, D. Werschmoeller, X. Li, F.E. Pfefferkorn, N.A. Duffie, Pulsed laser polishing of micro-milled Ti6Al4V samples, Journal of Manufacturing Processes. 11 (2009) 74-81. doi:10.1016/j.jmapro.2009.10.001.

[34] S. Tao, B. Wu, Y. Zhou, Y. Gao, Thermal modeling and experimental study of infrared nanosecond laser ablation of silicon, Journal of Applied Physics. 106 (2009) 123507. doi:10.1063/1.3271413.

[35] Y. Cao, X. Zhao, Y.C. Shin, Analysis of nanosecond laser ablation of aluminum with and without phase 
explosion in air and water, Journal of Laser Applications. 25 (2013) 032002. doi:10.2351/1.4794032.

[36] N.M. Bulgakova, A.V. Bulgakov, Pulsed laser ablation of solids: transition from normal vaporization to phase explosion, Applied Physics A Materials Science \& Processing. 73 (2001) 199-208. doi:10.1007/s003390000686.

[37] Q. Lu, S.S. Mao, X. Mao, R.E. Russo, Theory analysis of wavelength dependence of laser-induced phase explosion of silicon, Journal of Applied Physics. 104 (2008) 083301. doi:10.1063/1.2978369.

[38] J.H. Yoo, S.H. Jeong, R. Greif, R.E. Russo, Explosive change in crater properties during high power nanosecond laser ablation of silicon, Journal of Applied Physics. 88 (2000) 1638. doi:10.1063/1.373865.

[39] G. Cristoforetti, S. Legnaioli, V. Palleschi, E. Tognoni, P.A. Benedetti, Observation of different mass removal regimes during the laser ablation of an aluminium target in air, J. Anal. At. Spectrom. 23 (2008) 1518-1528. doi:10.1039/B800517F.

[40] A. Gragossian, S.H. Tavassoli, B. Shokri, Laser ablation of aluminum from normal evaporation to phase explosion, Journal of Applied Physics. 105 (2009) 103304. doi:10.1063/1.3131689.

[41] C. Porneala, D.A. Willis, Observation of nanosecond laser-induced phase explosion in aluminum, Applied Physics Letters. 89 (2006). doi:10.1063/1.2393158.

[42] A. V Gusarov, I. Smurov, Near-surface laser-vapour coupling in nanosecond pulsed laser ablation, Journal of Physics D: Applied Physics. 36 (2003) 2962. http://stacks.iop.org/0022-3727/36/i=23/a=016.

[43] J.M. Fishburn, R.P. Mildren, D. Kapitan, M.J. Withford, D.J.W. Brown, J.A. Piper, Exploring the explosive ablation regime of metals in nanosecond micromachining, in: C.R. Phipps, M. Niino (Eds.), 2000: pp. 453460. doi:10.1117/12.376996.

[44] A. Miotello, R. Kelly, Laser-induced phase explosion: New physical problems when a condensed phase approaches the thermodynamic critical temperature, Applied Physics A: Materials Science and Processing. 69 (1999). doi:10.1007/s003399900296.

[45] J.H. Yoo, S.H. Jeong, X.L. Mao, R. Greif, R.E. Russo, Evidence for phase-explosion and generation of large particles during high power nanosecond laser ablation of silicon, Applied Physics Letters. 76 (2000) 783. doi:10.1063/1.125894.

[46] B. Wu, From supercritical hydrodynamic expansion to explosive phase change: Thermodynamic evolution of water during its interaction with high-intensity infrared nanosecond-pulsed laser, Journal of Applied Physics. 105 (2009) 053502. doi:10.1063/1.3082120.

[47] P.. Mannion, J. Magee, E. Coyne, G.. O'Connor, T.. Glynn, The effect of damage accumulation behaviour on ablation thresholds and damage morphology in ultrafast laser micro-machining of common metals in air, Applied Surface Science. 233 (2004) 275-287. doi:10.1016/j.apsusc.2004.03.229.

[48] S. Tao, Y. Zhou, B. Wu, Y. Gao, Infrared long nanosecond laser pulse ablation of silicon: Integrated twodimensional modeling and time-resolved experimental study, Applied Surface Science. 258 (2012) 77667773. doi:10.1016/j.apsusc.2012.04.141.

[49] W.T. Nichols, T. Sasaki, N. Koshizaki, Laser ablation of a platinum target in water. II. Ablation rate and nanoparticle size distributions, Journal of Applied Physics. 100 (2006) 114912. doi:10.1063/1.2390641.

[50] Y. Cao, Y.C. Shin, Multi-scale modeling of phase explosion in high fluence nanosecond laser ablation and clarification of ablation depth prediction criterion, Applied Surface Science. 357 (2015) 74-85. doi:doi:10.1016/j.apsusc.2015.08.251.

[51] V.P. Carey, Liquid Vapor Phase Change Phenomena: An Introduction to the Thermophysics of Vaporization and Condensation Processes in Heat Transfer Equipment, Second Edition, Taylor \& Francis, 2007.

[52] W.M. Steen, Laser Surface Treatment, in: Laser Material Processing, Springer London, London, 2003: pp. 227-278. doi:10.1007/978-1-4471-3752-8_7.

[53] S.C. Erickson, Properties of Pure Metals, in: Properties and Selection: Nonferrous Alloys and SpecialPurpose Materials, Vol 2, ASM Handbook, ASM International, Materials Park, Ohio, USA, 1990: pp. 1099- 
1201.

[54] C.B. Alcock, V.P. Itkin, M.K. Horrigan, Vapour Pressure Equations for the Metallic Elements: 298-2500K, Canadian Metallurgical Quarterly. 23 (1984) 309-313. doi:10.1179/cmq.1984.23.3.309.

[55] V. Morel, A. Bultel, B.G. Chéron, The Critical Temperature of Aluminum, International Journal of Thermophysics. 30 (2009) 1853-1863. doi:10.1007/s10765-009-0671-6.

[56] eFunda: General Information on Element Copper, (n.d.). http://www.efunda.com/materials/elements/element_info.cfm?Element_ID $=\mathrm{Cu}$.

[57] D.R. Lide, Properties of the Elements and Inorganic Compounds; Vapor Pressure of the Metallic Elements, in: David R. Lide (Ed.), CRC Handbook of Chemistry and Physics, 84th Edition, CRC Press, Boca Raton, Florida, 2003.

[58] C. Garcia-Cordovilla, E. Louis, A. Pamies, The surface tension of liquid pure aluminium and aluminiummagnesium alloy, Journal of Materials Science. 21 (1986) 2787-2792. doi:10.1007/BF00551490.

[59] J.M. Molina, R. Voytovych, E. Louis, N. Eustathopoulos, The surface tension of liquid aluminium in high vacuum: The role of surface condition, International Journal of Adhesion and Adhesives. 27 (2007) 394401. doi:10.1016/j.ijadhadh.2006.09.006.

[60] J.G. Kaufman, B.E. Boardman, D.R. Gerty, B. Hindin, S. McComick, P.J. Sikorsky, et al., ASM Ready Reference: Properties and Units for Engineering Alloys, ASM International, 1997. https://books.google.com/books?id=UAhr9fdHYLMC.

[61] T. Matsumoto, H. Fujii, T. Ueda, M. Kamai, K. Nogi, Measurement of surface tension of molten copper using the free-fall oscillating drop method, Measurement Science and Technology. 16 (2005) 432-437. doi:10.1088/0957-0233/16/2/014.

[62] D.A. Harrison, D. Yan, S. Blairs, The surface tension of liquid copper, The Journal of Chemical Thermodynamics. 9 (1977) 1111-1119. doi:10.1016/0021-9614(77)90112-4.

[63] J.M. Fishburn, D.W. Coutts, M.J. Withford, J.A. Piper, Phase explosion thresholds in metals ablated under pulsed nanosecond radiation, in: Australasian Conference on Optics, Lasers and Spectroscopy, Brisbane, Queenslan, Austrila, 2001: p. 127.

[64] S.H. Tavassoli, M. Khalaji, Laser ablation of preheated copper samples, Journal of Applied Physics. 103 (2008) 083118. doi:10.1063/1.2907955.

[65] C. Liu, X.L. Mao, S.S. Mao, X. Zeng, R. Greif, R.E. Russo, Nanosecond and Femtosecond Laser Ablation of Brass: Particulate and ICPMS Measurements, Analytical Chemistry. 76 (2004) 379-383. doi:10.1021/ac035040a.

[66] P. Lorazo, L.J. Lewis, M. Meunier, Short-Pulse Laser Ablation of Solids: From Phase Explosion to Fragmentation, Physical Review Letters. 91 (2003) 225502. doi:10.1103/PhysRevLett.91.225502. 